\title{
The actin-binding protein EPS8 binds VE-cadherin and modulates YAP localization and signaling
}

\author{
Costanza Giampietro, 1,6 Andrea Disanza, ${ }^{1}$ Luca Bravi, ${ }^{1}$ Miriam Barrios-Rodiles, ${ }^{2}$ Monica Corada, ${ }^{1}$ Emanuela Frittoli, \\ Cecilia Savorani, ${ }^{1}$ Maria Grazia Lampugnani, ${ }^{1,7}$ Barbara Boggetti, ${ }^{4}$ Carien Niessen ${ }^{4}$ Jeff L. Wrana, ${ }^{2,3}$ \\ Giorgio Scita, ${ }^{1,5}$ and Elisabetta Dejana ${ }^{1,6,8}$

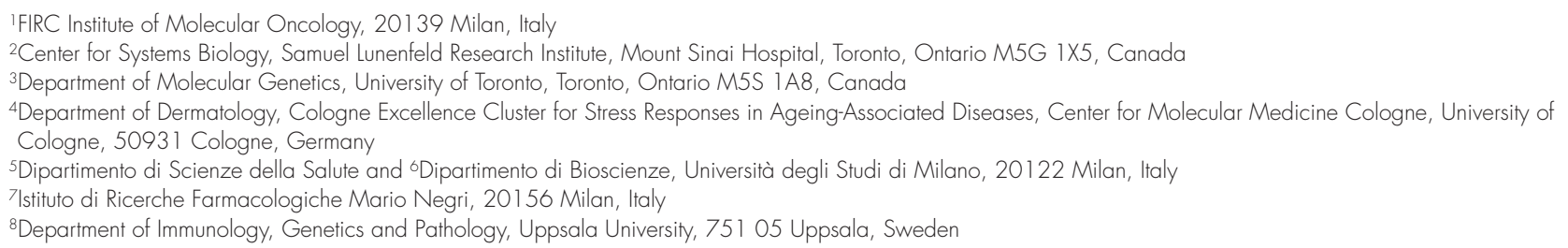

IFIRC Institute of Molecular Oncology, 20139 Milan, Italy

${ }^{2}$ Center for Systems Biology, Samuel Lunenfeld Research Institute, Mount Sinai Hospital, Toronto, Ontario M5G 1X5, Canada

3Department of Molecular Genetics, University of Toronto, Toronto, Ontario M5S 1A8, Canada

${ }^{4}$ Department of Dermatology, Cologne Excellence Cluster for Stress Responses in Ageing-Associated Diseases, Center for Molecular Medicine Cologne, University of

Cologne, 50931 Cologne, Germany

${ }^{5}$ Dipartimento di Scienze della Salute and 'Dipartimento di Bioscienze, Università degli Studi di Milano, 20122 Milan, Italy

7Istituto di Ricerche Farmacologiche Mario Negri, 20156 Milan, Italy

${ }^{8}$ Department of Immunology, Genetics and Pathology, Uppsala University, 75105 Uppsala, Sweden

Vascular endothelial (VE)-cadherin transfers intracellular signals contributing to vascular hemostasis. Signaling through VE-cadherin requires association and activity of different intracellular partners. Yes-associated protein (YAP)/TAZ transcriptional cofactors are important regulators of cell growth and organ size. We show that EPS8, a signaling adapter regulating actin dynamics, is a novel partner of VE-cadherin and is able to modulate YAP activity. By biochemical and imaging approaches, we demonstrate that EPS8 associates with the VE-cadherin complex of remodeling junctions promoting YAP translocation to the nucleus and transcriptional activation. Conversely, in stabilized junctions, 14-3-3-YAP associates with the VE-cadherin complex, whereas Eps8 is excluded. Junctional association of YAP inhibits nuclear translocation and inactivates its transcriptional activity both in vitro and in vivo in Eps8-null mice. The absence of Eps8 also increases vascular permeability in vivo, but did not induce other major vascular defects. Collectively, we identified novel components of the adherens junction complex, and we introduce a novel molecular mechanism through which the VE-cadherin complex controls YAP transcriptional activity.

\section{Introduction}

Endothelial cells (ECs) form the inner lining of blood vessels, and one of their most important properties is to separate blood from underlying tissues. Their role as a selective permeability barrier is mainly achieved through the coordinated opening and closure of cell-to-cell junctions. In addition to maintaining adhesion between neighboring cells, junctions play crucial roles in transducing chemical and mechanical signals that regulate contact-induced inhibition of cell growth, apoptosis, gene expression, and vessel formation and stability (Vandenbroucke et al., 2008; Giampietro et al., 2012; Giannotta et al., 2013).

EC homotypic adhesion is mainly controlled by two types of adhesive structures: tight and adherens junctions (AJs; McCrea et al., 2009; Vestweber et al., 2009; Giannotta et al., 2013). The key component of AJs is transmembrane vascular endothelial (VE)-cadherin, an endothelial-specific member of the cadherin family. VE-cadherin is physically connected to a large

Correspondence to Costanza Giampietro: costanza.giampietro@unimi.it; or Elisabetta Dejana: elisabetta.dejana@ifom.eu; or Giorgio Scita: giorgio.scita@ ifom.eu

Abbreviations used in this paper: $\mathrm{A}$, adherens junction; $\mathrm{EC}$, endothelial cell; IF, immunofluorescence; IP, immunoprecipitation; Ni-NTA, nickel-nitrilotriacetic acid; qRT-PCR, quantitative RT-PCR; RL, Renilla luciferase; VE, vascular endothelial; WB, Western blot; WT, wild type; YAP, Yes-associated protein. number of intracellular partners that mediate its anchorage to the actin cytoskeleton and the transfer of signals essential to modulate endothelial functions (Vestweber et al., 2009; Dejana and Giampietro, 2012). Not surprisingly, changes in the structure and composition of AJs have profound effects on vascular permeability as well as on the overall vascular homeostasis (Vestweber et al., 2010).

Junctions are dynamic structures whose regulation and structural changes strongly impact adhesion strength and tissue plasticity. ECs from different types of vessels and also from different organs show differences in junction composition and organization (Orsenigo et al., 2012; Kluger et al., 2013).

Recent studies revealed that the cotranscriptional regulator YAP (Yes-associated protein), originally characterized as the molecular target of the size-controlling Hippo pathway (Varelas, 2014), is a key relay for the transmission of mechanical inputs into gene transcriptional programs (Dupont et al., 2011). Indeed, multiple signaling pathways integrating biophysical

(C) 2015 Giampietro et al. This article is distributed under the terms of an AttributionNoncommercial-Share Alike-No Mirror Sites license for the first six months after the publication date (see http://www.rupress.org/terms). After six months it is available under a Creative Commons License (Attribution-Noncommercial-Share Alike 3.0 Unported license, as described at http://creativecommons.org/licenses/by-nc-sa/3.0/). 
A
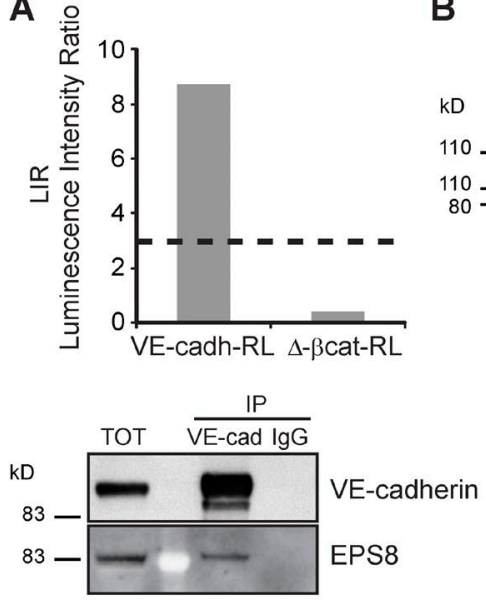

D VE-cadherin
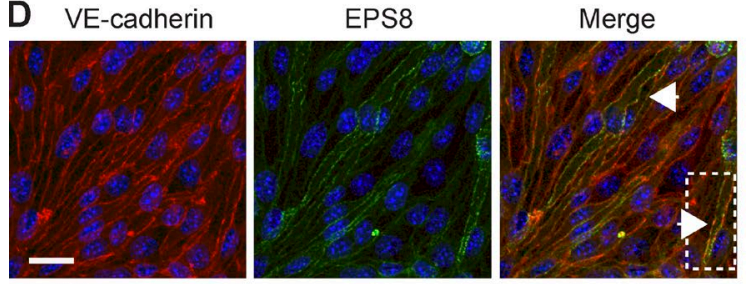

E

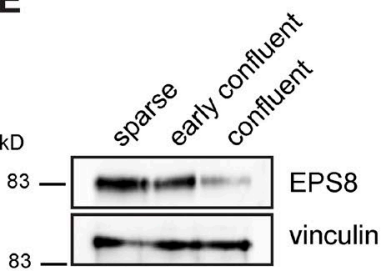

B sparse

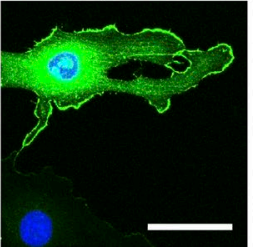

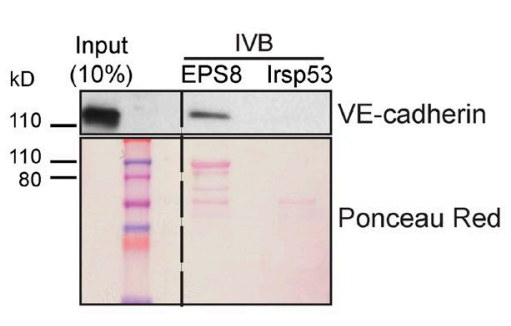

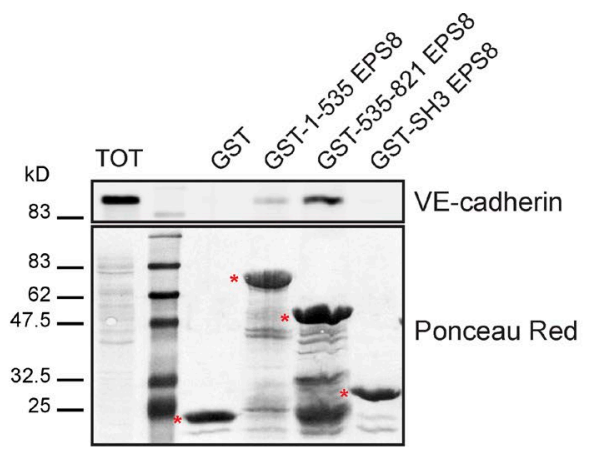

C

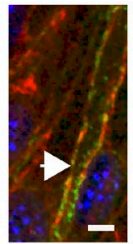

kD

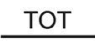

TOT IPs
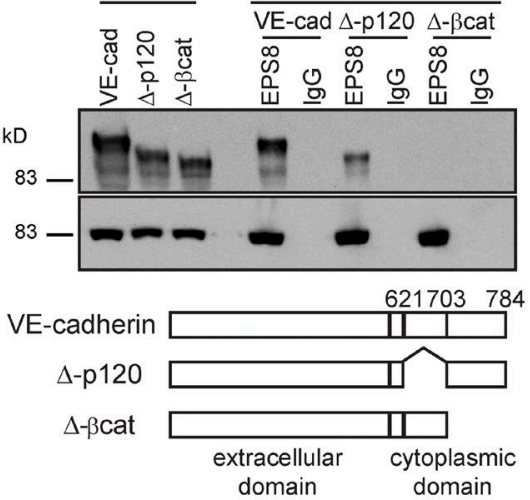

VE-cadherin EPS8

nt
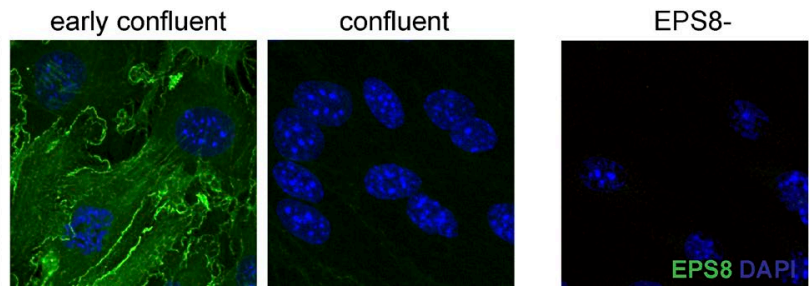

Figure 1. EPS8 is a novel component of AJ complexes in ECs. (A) Detection of full-length VE-cadherin-EPS8 interaction by LUMIER (top); the data are representative of five independent experiments. Association of EPS8 to full-length VE-cadherin (bottom). Coimmunoprecipitation and Western blot of endogenous VE-cadherin and EPS8 from extract of VE-Cadherin-positive ECs. The dashed line indicates an LIR of 3, a conservative LIR cutoff. (B) Analysis of VE-cadherin cytoplasmic tail and EPS8 full-length interaction in vitro by His-tag pull-down assay (left). Analysis of the interaction between VE-cadherin cytoplasmic tail and EPS8 deleted mutants (asterisks) in vitro by GST pull-down assay (right). (C) Association of EPS8 to VE-cadherin full-length (VE-cad), $\Delta$-p 120, and $\Delta$ - $\beta$ cat mutants upon transient expression in COS-1 cells. Cells were transfected with the indicated constructs, immunoprecipitated with specific EPS8 antibody or isotype control lgG, and blotted as indicated. (D) IF microscopy of VE-cadherin-positive ECs in early confluent (24 h) conditions. Cells were double stained with anti-VE-cadherin (red) and anti-EPS8 (green) antibodies. Junctional EPS8 colocalizing with VE-cadherin (arrows) was detected. The dashed outline indicates the magnified area to the right. (E) WB analysis (left) of EPS8 expression levels in various stages of confluence conditions (see Materials and methods section). Vinculin is the loading control. IF microscopy (right) of WT lung-derived ECs in various stages of confluence conditions. The IF of Eps8- ECs confirmed the specificity of the staining. Cells were stained with anti-EPS8 antibody (green) and DAPI (blue). Bars: (D and E) 20 pm; (magnification) $10 \mu \mathrm{m}$. IVB, in vitro binding; TOT, total cell lysate.

and biochemical cues converge to regulate the activity of YAP (Morgan et al., 2013). YAP, in turn, is essential to modulate cell proliferation and differentiation, apoptosis, organ size, and morphogenesis of various tissues (Zhao et al., 2011). In epithelial tissues, for example, YAP has been shown to be regulated by the formation of cell-cell contacts, to be required for contact inhibition of cell proliferation (Zhao et al., 2007), and to respond to mechanical perturbation of the epithelial sheet (Aragona et al., 2013). In all these situations, actin cytoskeletal-based mechanical forces have been shown to be the overarching regulator of the activity of YAP and its related molecule TAZ, setting responsiveness to a variety of key signaling axes, including the Hippo, WNT, and G protein-coupled receptor pathways. Notably, Yap $^{-/-}$mice display an early embryonic lethal phenotype resulting from defects in yolk sac vasculogenesis, chorioallantoic fusion, and embryonic axis elongation (Morin-Kensicki et al., 2006), suggesting a role of this protein also in the control of endothelial morphogenetic processes. The molecular determinants through which ECs control YAP regulation remain, however, largely unexplored.

The EGF receptor kinase substrate 8 (EPS8) is a signaling adapter protein involved in the transduction of signal from RAS to RAC (Scita et al., 1999). EPS8 also directly binds to actin filaments controlling the rate of polymerization/depolymerization by capping the fast-growing ends of filaments (Croce et al., 2004; Disanza et al., 2004, 2006; Hertzog et al., 2010). Consistently, EPS8, in vivo, is required for optimal actin-based motility impacting migratory properties of different cells (Frittoli et al., 2011). Furthermore, EPS8 regulates the proper architectural organization of actin-based structures, including intestinal microvilli and stereocilia (Disanza et al., 2006; Hertzog et al., 2010; Tocchetti et al., 2010; Manor et al., 2011). One 
A

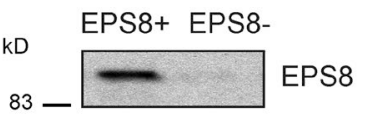

C

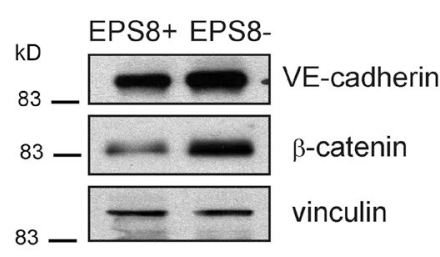

E

EPS8+ EPS8-

IP: VE-cad IgG VE-cad IgG

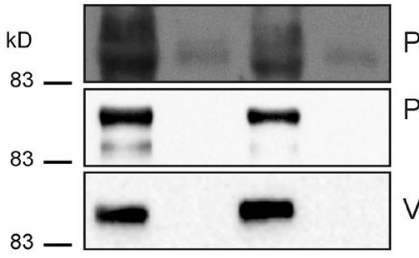

P4D1 ubiquitin

Phospho VE-cadherin

(Ser665)

VE-cadherin
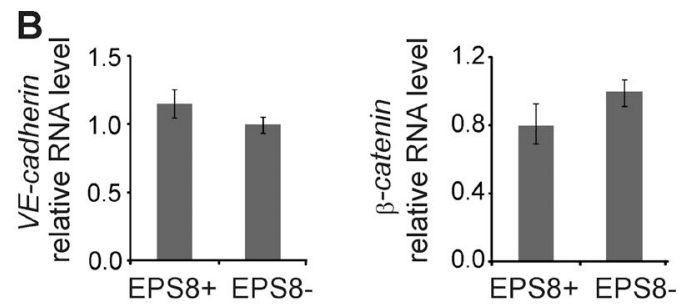

D
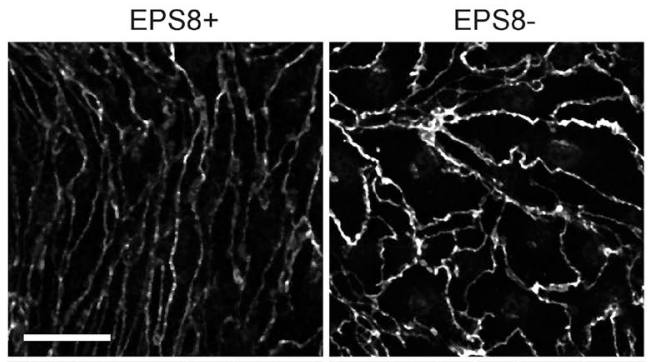

VE-cadherin
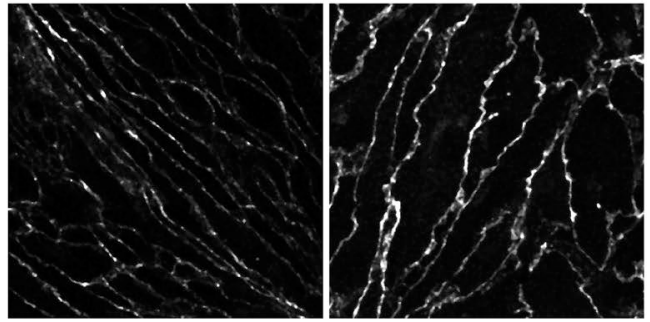

Figure 2. Effect of EPS8 expression on AJ organization and dynamics. (A) WB (left) and qRT-PCR (right) analysis of EPS8 reconstitution in eps8-/- lungderived ECs. Cells were infected with either WT EPS8-GFP (EPS8+) or control GFP (EPS8-) lentiviral vectors. The gene expression level has been represented as fold changes \pm SEM of three independent experiments. $(B)$ qRT-PCR analysis of VE-cadherin $(P=0.06)$ and $\beta$-catenin $(P=0.07)$ in EPS8+ and EPS8ECs. For each tested gene, the expression level has been represented as fold changes \pm SEM of four independent experiments. (C) WB analysis (left) of VE-cadherin and $\beta$-catenin expression in extracts of EPS8+ and EPS8- ECs in confluent condition. Vinculin is shown as the loading control. The graph (right) represents the WB quantification. Columns are means \pm SEM of three independent experiments. The dashed line indicates that relative levels for the Eps $8^{+}$ cells were set to 1. (D) IF microscopy of confluent EPS8+ and EPS8- cells stained with anti-VE-cadherin and - $\beta$-catenin antibody. An increase of junctional proteins' distribution is observed in the absence of EPS8. Bar, $20 \mu \mathrm{m}$. (E) IP of VE-cadherin from EPS8+ and EPS8- cell extracts followed by WB analysis of phosphorylation on serine 665 residue and ubiquitin level (top). Quantifications of three independent experiments are shown as means \pm SD at the bottom. *, $\mathrm{P}<0.05 ; * *, \mathrm{P}<0.01$.

additional cellular process in which EPS8 is implicated is the regulation of intracellular trafficking of various membrane receptors (Lanzetti et al., 2000; Di Fiore and Scita, 2002; Auciello et al., 2013). EPS8 exerts this function either through its direct interaction with the GTPase-activating protein, RN-tre, which controls the activity of RAB5, a master regulator of early endosomes (Lanzetti et al., 2000; Di Fiore and Scita, 2002), or by interacting with the clathrin-mediated endocytosis machinery (Taylor et al., 2012; Auciello et al., 2013).

Here, we identified EPS8 as a novel partner of VEcadherin at AJs. We also found that EPS8 regulates the dynamic organization of endothelial junctions and the transduction of intracellular signals by tuning YAP transcriptional activity.

\section{Results}

\section{EPSB is a novel component of \\ A complexes}

To identify novel components of the VE-cadherin signaling complex, we set up a LUMIER (luminescence-based mammalian interactome mapping) automated high throughput screening. This approach is designed for the systematic mapping of dynamic protein-protein interaction networks in mammalian cells (Barrios-Rodiles et al., 2005). Full-length VE-cadherin and $\Delta$ - $\beta$ cat mutant, devoid of the $\mathrm{C}$-terminal interaction domain with $\beta$-catenin (Fig. 1 C, bottom; Navarro et al., 1995), were fused to Renilla luciferase (RL) and coexpressed with a library 
A
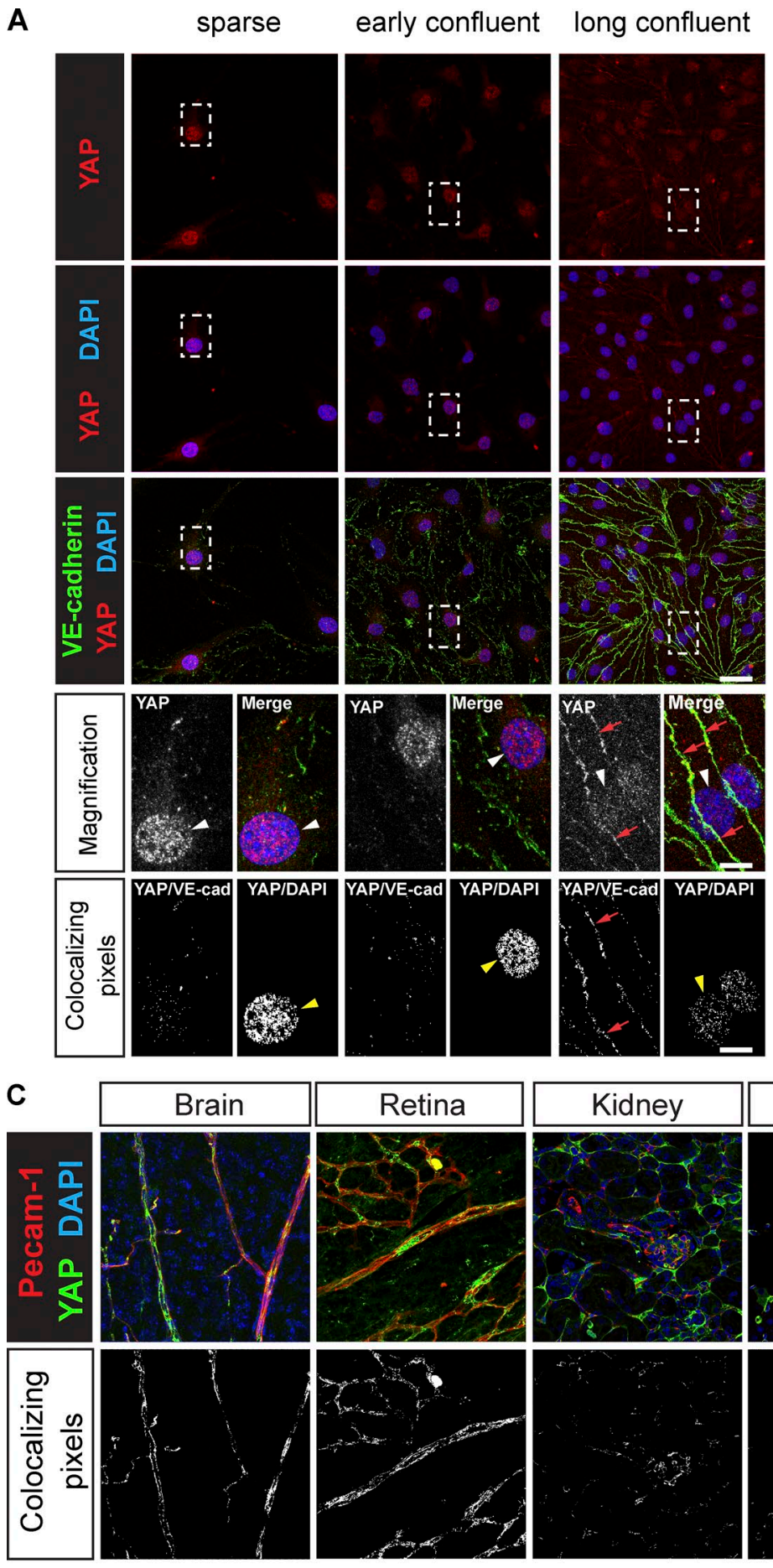

B

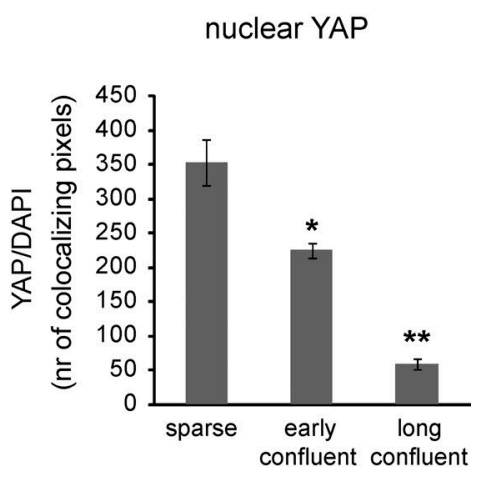

junctional YAP

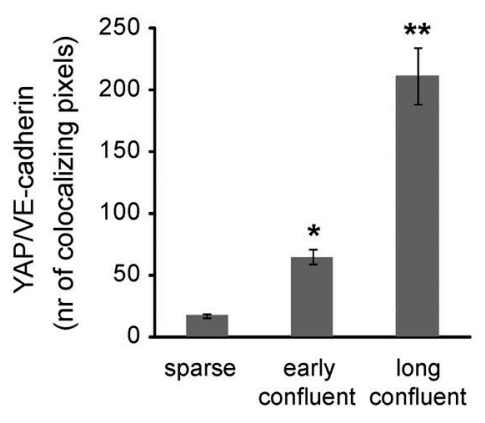

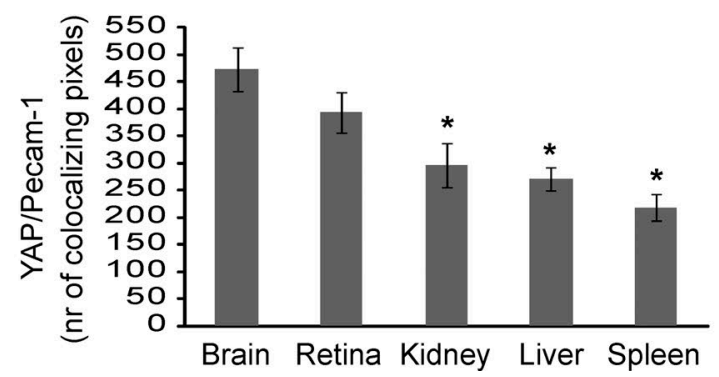


of individual Flag-tagged partners in mammalian HEK293T cells. We next assessed the association of VE-cadherin wildtype (WT) and mutant proteins with Flag-tagged proteins by performing a luciferase assay on anti-Flag immunoprecipitates. Among a variety of identified interactors, we focused our investigation on EPS8, which bound full-length VE-cadherin-RL, but not $\Delta$ - $\beta$ cat-RL mutant (Fig. 1 A, top). We validated the physiological relevance of this association by coimmunoprecipitation of endogenous proteins from whole WT EC extracts (Fig. $1 \mathrm{~A}$, bottom). In vitro pull-down experiments using recombinant purified proteins indicated that EPS8 binds directly, through its $\mathrm{C}$-terminal domain, to the $\mathrm{C}$-terminal region of the cytoplasmic tail of VE-cadherin (Fig. 1 B). Coimmunoprecipitation experiments between EPS8 and a set of deletion mutants of VE-cadherin corroborated the results obtained with LUMIER. EPS8 efficiently bound to WT and a VE-cadherin mutant devoid of the interaction domain with p120 $(\Delta-$ p120; Lampugnani et al., 1997) but failed to associate with a $\Delta$ - $\beta$ cat VE-cadherin, which binds neither endogenous $\beta$-catenin nor $\alpha$-catenin (Fig. $1 \mathrm{C}$, top), indicating that the region spanning amino acids 703-784 of VE-cadherin is indispensable for this interaction. Confocal analyses of early confluent WT (24 h) ECs showed that EPS8 was enriched along cell-to-cell junctions and largely colocalized with VE-cadherin (Fig. 1 D, arrows and magnification). Notably, EPS8 expression and junctional localization were down-regulated in long confluent WT EC culture (72 h; Fig. 1 E). To confirm the specificity of the staining, we also performed immunofluorescence (IF) analysis on early confluent Eps8-null ECs (EPS8-) that consistently did not reveal any signal.

\section{EPSB increases the dynamic turnover of VE-cadherin}

To gain functional cues into the role of EPS8 in ECs, we derived ECs from lungs of eps8-null mice, which were subsequently reconstituted with either WT EPS8-EGFP (EPS8 ${ }^{+}$cells) or control EGFP (EPS8- cells) lentiviral vectors (Fig. 2 A; Menna et al., 2009). EC populations are characterized by high phenotypic heterogeneity (Ribatti et al., 2002); thus, we decided to use this approach to work with the same cell line, differing only for the expression of EPS8. The level of the EPS8 in EPS8 ${ }^{+}$ECs is $\sim 30 \%$ more than WT ECs (Fig. S1 A), but this does not significantly impact the molecular mechanisms studied, as shown in the following paragraph. In the absence of EPS8, the major components of the AJ complexes were up-regulated (Fig. 2 C) at junctions (Fig. 2 D), but the corresponding mRNAs remained unchanged (Fig. 2 B), suggesting a posttranscriptional effect. VE-cadherin is internalized through clathrin-mediated pathways in a process that requires VE-cadherin phosphorylation and ubiquitination (Gavard and Gutkind, 2006; Orsenigo et al., 2012). Expression of EPS8 increased VE-cadherin phosphorylation on serine 665 and ubiquitination (Fig. 2 E), suggesting enhanced VE-cadherin turnover.
EPSB and YAP localization at junctions is mutually exclusive

Cadherin complexes can either repress or stimulate gene transcription. Considering the role of EPS8 in cytoskeletal organization, we asked whether the association of EPS8 with VE-cadherin might influence signaling pathways modulated by actin remodeling. We focused on the transcriptional cofactor YAP because its nuclear-cytoplasmic shuttling and activity may be regulated by cadherin-mediated cell-cell contacts (Kim et al., 2011) and cytoskeletal-dependent mechanical forces (Dupont et al., 2011). YAP was, as shown in other cell types (Aragona et al., 2013), almost entirely localized in the nucleus of sparse WT ECs (Fig. 3, A and B). Conversely, in early confluent and long confluent WT endothelial monolayers, it was gradually excluded from the nuclei (Fig. 3, A and B) and redistributed along cell-to-cell junctions, where it colocalized with VE-cadherin (Fig. 3, A and B). To detect the junctional localization of YAP, cells have been fixed with $1 \%$ PFA in $2.5-\mathrm{mM}$ triethanolamine (see Materials and methods section IF microscopy). In vivo, YAP localization at cell-cell junctions was detectable in the vessels of the brain and the retina of neonatal mice. Conversely, in vessels of other organs such as spleen, kidney, and liver, where the junctions are loose, YAP expression at junctions was decreased (Fig. 3 C).

Overall, these in vitro and in vivo data suggest that EPS8 and YAP localize at junctions in a mutually exclusive and temporally distinct manner. In particular, EPS8 is a marker of early and dynamic junctions, whereas YAP is mostly recruited at stable junctions.

We then asked whether EPS8 might regulate YAP signaling. To this end, we compared EPS8 ${ }^{+}$to EPS8 ${ }^{-}$ECs in early confluent conditions when EPS8 is prominently localized at intercellular junctions in WT ECs (Fig. 1 E). Under these conditions, we found that in EPS8- ECs, YAP phosphorylation on serine 127 , an inhibitory posttranslational modification (Zhao et al., 2007), was increased (Fig. 4 A), and YAP nuclear fraction was diminished (Fig. 4, B and C) as well as the expression of its target genes (Fig. 4 D), whose mRNAs are reduced by $\sim 10$ fold. Of note, as reported in Fig. S1 B, the expression of YAP target genes was significantly high in sparse conditions, and it was not affected by the presence or absence of EPS8. YAP target gene expression was strongly reduced by early cell confluence as previously published (Schlegelmilch et al., 2011), but the reductions were significantly less in the absence of EPS8, supporting the idea that EPS8 sustained YAP transcriptional activity only when it was localized at AJs (Fig. 1 E).

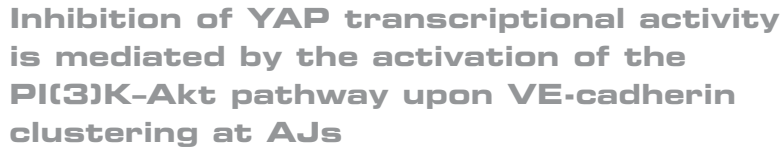

A phosphorylation-dependent "shuttling" between the cytoplasm and the nucleus regulates the transcriptional activity of

Figure 3. YAP is localized at junctions in long confluent monolayers in vitro and in the more stabilized vessels in vivo. (A, top) Confocal microscopy analysis of YAP (red) colocalization with VE-cadherin (green) and nuclei (DAPI, blue) in WT lung-derived ECs at different stages of confluence conditions (see Cell lines in the Materials and methods section). Bar, 20 mm. (A, bottom) Pixels presenting the colocalization of VE-cadherin and YAP are highlighted in white. Bars, $10 \mu \mathrm{m}$. Nuclei are highlighted with arrowheads, and junctions are highlighted with arrows. (B) Quantification of the number of colocalizing pixels between YAP and DAPI (top) or VE-cadherin (bottom) is shown. Data are means \pm SEM of three independent experiments. ${ }^{*}, P<0.05 ;{ }^{* *}, \mathrm{P}<0.01$. (C) Confocal microscopy analysis of Pecam-1 (red) and YAP (green) localization in different organs of P9 mice (top). Bars, 50 um. Pixels presenting the colocalization of Pecam-1 and YAP are highlighted in white. Quantification of the number of colocalizing pixels between YAP and Pecam-1 (bottom). Data are means \pm SEM of five mice analyzed. ${ }^{*}, \mathrm{P}<0.05$ for retina, kidney, liver, and spleen versus brain. 
A

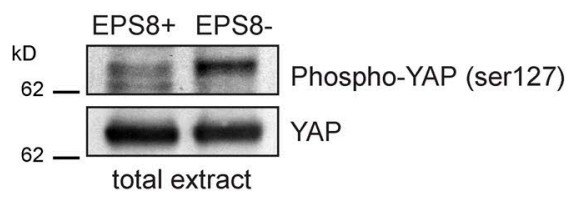

C

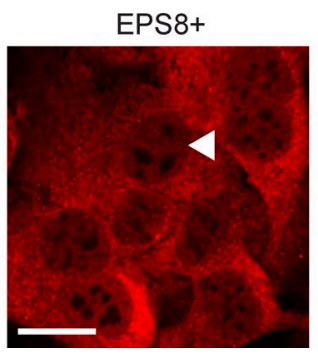

B

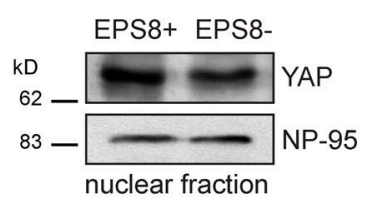

D

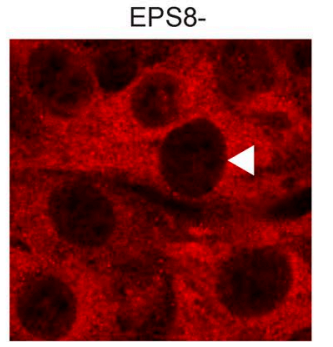

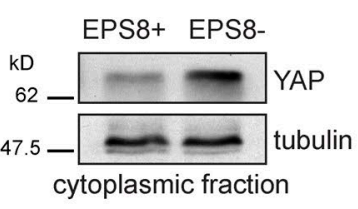

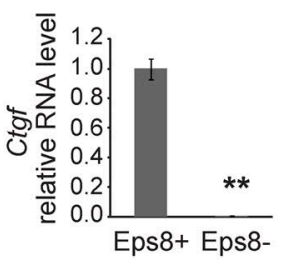

- cytoplasm $\approx$ nucleus
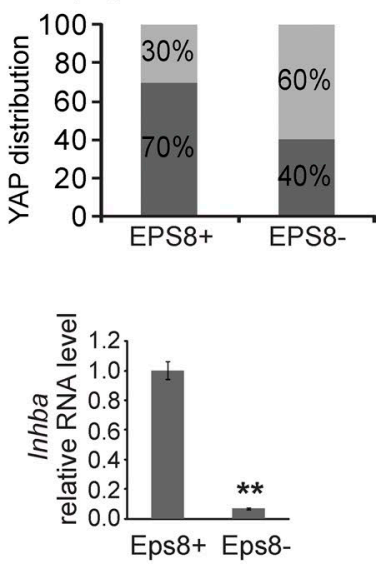

Figure 4. EPS8 expression modulates YAP phosphorylation, localization, and transcriptional activity in confluent ECs. (A) YAP phosphorylation (PhosphoYAP serine 127) in confluent EPS8+ and EPS8- cells. Total cell lysates were analyzed by WB for phosphorylated and total YAP expression by using specific antibodies. (B, left) WB analysis of nuclear/cytoplasmic distribution of YAP in confluent EPS8+ and EPS8- cells. On EPS8 removal (right), YAP shifted from a preferentially nuclear to a preferentially cytoplasmic localization. NP-95 and tubulin were used as nuclear and cytoplasmic markers, respectively. The graph on the right represents the quantification of four independent experiments. (C) IF microscopy of YAP (red) nuclear localization (arrowheads) in EPS8+ and EPS8- ECs. Bar, 20 m. (D) qRT-PCR analysis of connective tissue growth factor (Ctgf), Cysteine-rich angiogenic inducer 61 (Cyr61), and Inhibin $\beta$ A (Inhba) in EPS8 ${ }^{+}$and EPS8- ECs to measure YAP transcriptional activity. The absence of EPS8 strongly inhibited YAP transcriptional activity. For each tested gene, the expression level has been represented as fold changes \pm SEM of three independent experiments. ${ }^{*}, \mathrm{P}<0.01$.

YAP (Varelas, 2014). We found that VE-cadherin expression and clustering was important to limit YAP transcriptional activity. As reported in Fig. S2 A, and consistent with previously published literature (Choi et al., 2015), the up-regulation of YAP target genes was severely inhibited in VE-cadherin-positive as compared with VE-cadherin-null ECs in confluent conditions. VE-cadherin clustering is known to activate Akt through PI(3) $\mathrm{K}$ (Carmeliet et al., 1999; Taddei et al., 2008). Akt, in turn, was shown to be able to phosphorylate YAP in serine 127 in vitro and in ECs (Basu et al., 2003; Choi et al., 2015). We therefore investigated whether EPS8 localization at AJs might affect Akt activation and Akt-dependent YAP phosphorylation. Both Akt and YAP phosphorylation were increased in EPS8- cells as compared with EPS8+ cells (Fig. 5 A). Pharmacological inhibition of PI(3)K with LY294002 reduced both Akt and YAP phosphorylation in EPS8 ${ }^{+}$and EPS8 ${ }^{-}$cells to a comparable level. LY294002 treatment also restored the expression of YAP-dependent genes in EPS8- ECs to levels similar to those observed in EPS8+ cells (Fig. 5 B). Conversely, the ectopic expression of myr-Akt, a constitutively active form of Akt (Brown et al., 2005), increased the phosphorylation of YAP in EPS8 ${ }^{+}$but not in EPS8- ECs. This lack of effect is likely a result of an already high basal AKT phosphorylation in the latter cell type (Fig. 5 C). myr-Akt infection was also able to reduce YAP target gene expression in $\mathrm{EPS}^{+}$but not EPS8- ECs (Fig. 5 D).

In epithelial cells, YAP transcriptional activity is reduced by the organization of E-cadherin-based AJs through the activation of the Hippo signaling pathway (Kim et al., 2011) and by the activity of small GTPases that act primarily through regulation of the actin cytoskeleton (Dupont et al., 2011). Of note, in ECs small GTPase activity is controlled by VE-cadherin clustering (Pannekoek et al., 2011; Giannotta et al., 2013; Goddard and Iruela-Arispe, 2013). However, neither the Hippo pathway (Fig. S3 A) nor the activity of RHO (Fig. S3 B) and RAC1 (Fig. S3 C) was altered by removal of EPS8.
Collectively, these data suggest that in ECs inhibition of $\mathrm{PI}(3) \mathrm{K}-\mathrm{Akt}$ is the prominent pathway through which EPS8 increases YAP activity.

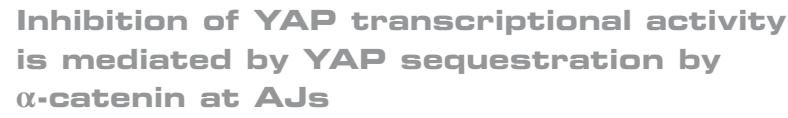

The association of YAP with $\alpha$-catenin limits its transcriptional activity (Silvis et al., 2011). $\alpha$-Catenin sequesters YAP in the cytoplasm, thus preventing its dephosphorylation and nuclear translocation (Schlegelmilch et al., 2011; Silvis et al., 2011). We investigated whether EPS8 might play a role in the regulation of this pathway. An endogenous YAP- $\alpha$-catenin complex could be detected by coimmunoprecipitation only in EPS8-, but not in EPS8 ${ }^{+}$ECs (Fig. 5 E). This complex associated with VE-cadherin as revealed by coimmunoprecipitation experiments (Fig. 5 F), and $\alpha$-catenin was required for YAP-VEcadherin interaction. Notably, removal of the VE-cadherin binding surface for $\beta$-catenin, which is required for $\alpha$-catenin binding but not for $\mathrm{p} 120$, reduced, as expected, YAP phosphorylation (Fig. S4 A), prevented YAP binding to VE-cadherin (Fig. S4 B), and enhanced YAP transcriptional activity (Fig. S4 C). Finally, silencing of $\alpha$-catenin impaired YAP localization at AJs (Fig. 5 F), reduced YAP phosphorylation (Fig. 5 G), and increased its transcriptional activity (Fig. $5 \mathrm{H}$ ) in a way more marked in the absence than in the presence of EPS8. These results suggest that EPS8 restrains the interaction of YAP with $\alpha$-catenin. As a consequence, upon removal of EPS8, YAP is more efficiently sequestered into junctional complexes that prevent its nuclear translocation and transcriptional activity. Of note, and in line with what has been previously published (Schlegelmilch et al., 2011; Silvis et al., 2011), we were unable to detect a complex between TAZ, the related protein of YAP, and $\alpha$-catenin, suggesting that TAZ activity may be regulated by different mechanisms, but specific studies are required to test this hypothesis. 


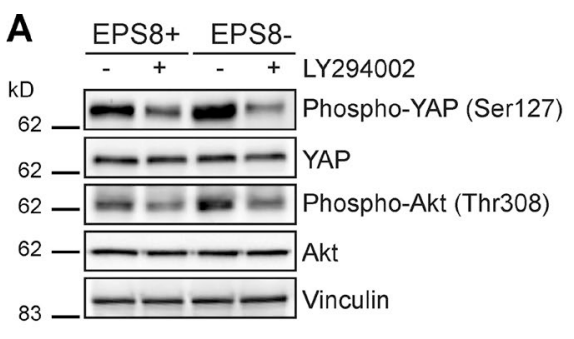

C

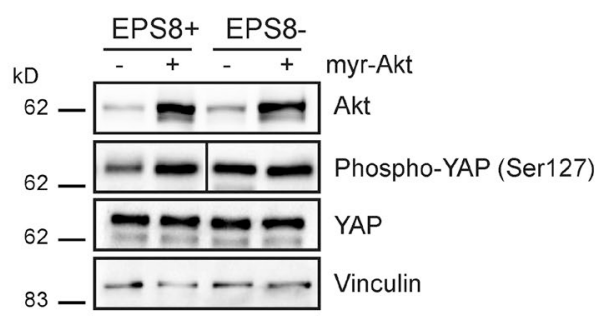

E
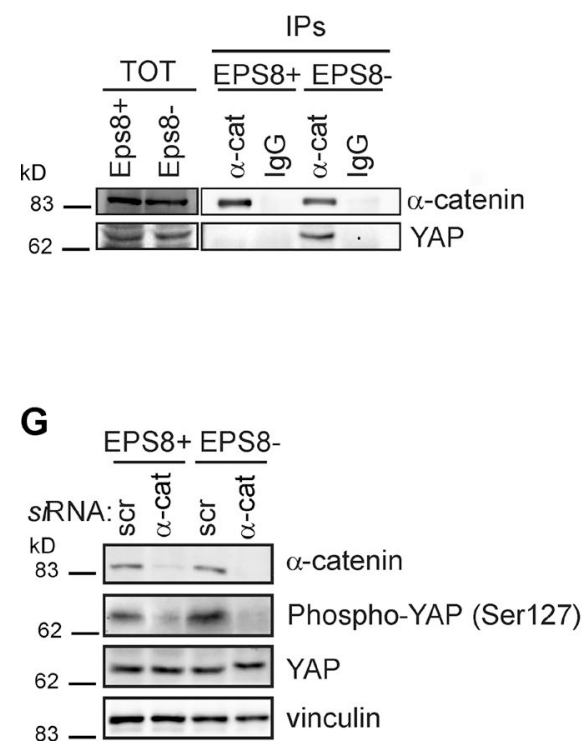
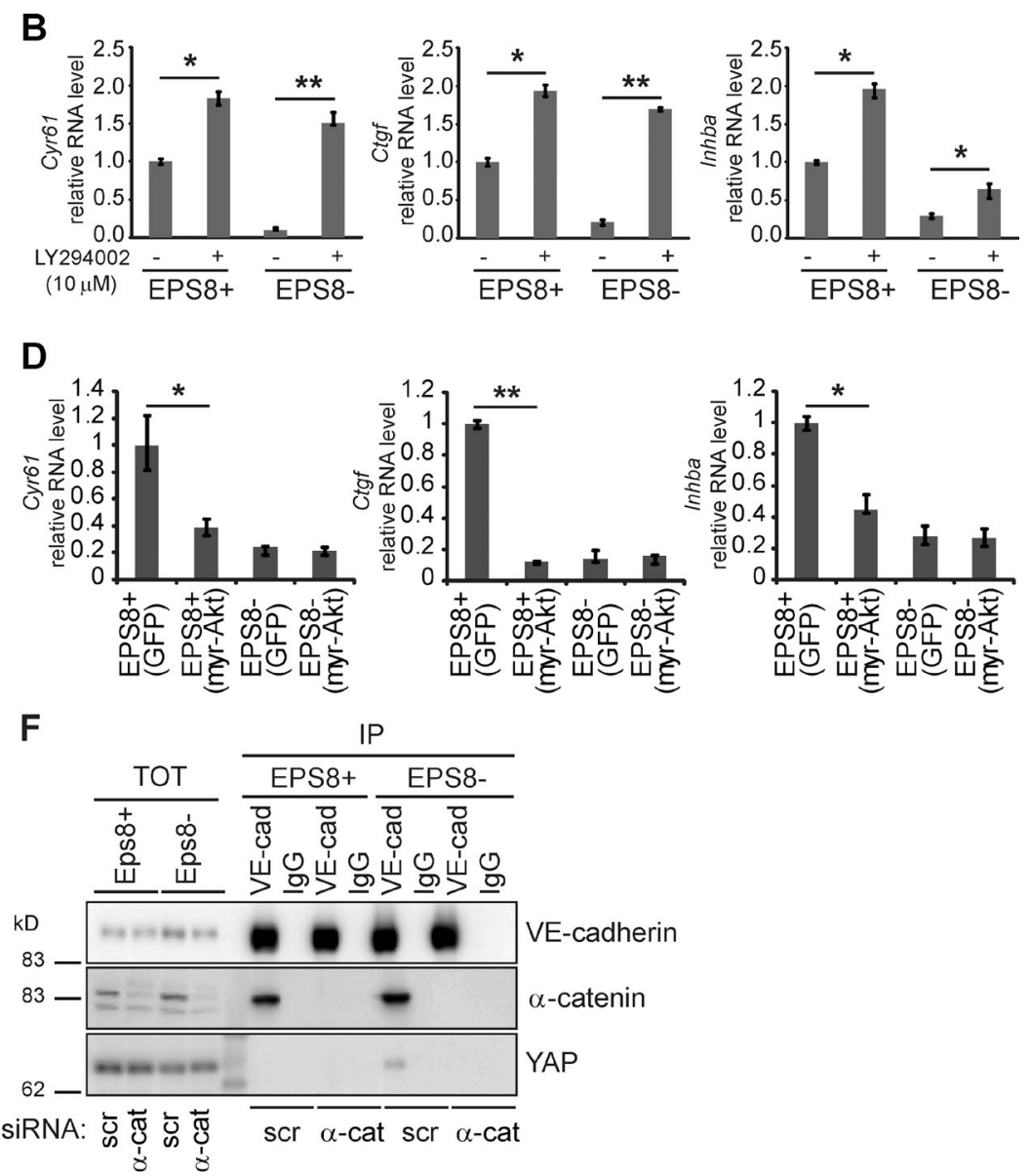

H
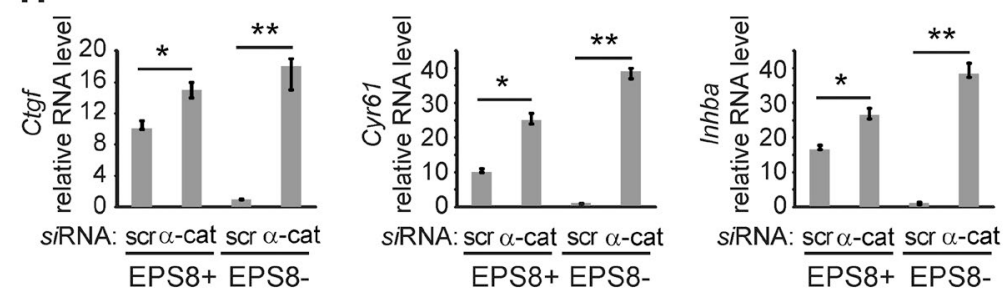

Figure 5. YAP phosphorylation and transcriptional activity are regulated through differential $\mathrm{PI}(3) \mathrm{K}$-Akt pathway activation and binding to $\alpha$-catenin. (A) WB analysis of YAP and Akt phosphorylation upon inhibition of the PI(3)K-Akt pathway. Cells were grown to $90 \%$ confluence, starved for $24 \mathrm{~h}$, and incubated overnight in complete medium with the PI(3)K inhibitor LY294002 (10 $\mu \mathrm{M})$. Vinculin is shown as a loading control. (B) qRT-PCR analysis of Cyr6 1, Ctgf, and Inhba to measure YAP transcriptional activity upon inhibition of PI(3)K in EPS8+ and EPS8- ECs. (C) WB analysis of YAP phosphorylation upon overexpression of constitutively active Akt (myr-Akt) in EPS8+ and EPS8- ECs. Vinculin is shown as a loading control. (D) qRT-PCR analysis of Cyr61, Ctgf, and Inhba to measure YAP transcriptional activity upon myr-Akt expression in EPS8+ and EPS8- ECs. (E) Coimmunoprecipitation and WB analysis of the YAP- $\alpha$-catenin complex in EPS8+ and EPS8- ECs. (F) IP of VE-cadherin and WB analysis of the YAP- $\alpha$-catenin complex localization at cell-cell contacts in EPS8+ and EPS8- cells upon specific siRNA of $\alpha$-catenin. (G) WB analysis of YAP phosphorylation upon specific siRNA of $\alpha$-catenin in EPS8 ${ }^{+}$and EPS8- ECs. Vinculin is shown as a loading control. (H) qRT-PCR analysis of Ctgf, Cyr61, and Inhba to measure YAP transcriptional activity upon siRNA of $\alpha$-catenin. For each tested gene, the expression level has been represented as fold changes \pm SEM of three independent experiments. ${ }^{*}, P<0.05$; $* *, P<0.01$. TOT, total cell lysate.

\section{EPSB and the 14-3-3-YAP complex}

compete for binding to $\alpha$-catenin

A common mechanism of cytoplasmic retention of nuclear proteins is mediated by the binding of 14-3-3 proteins, which interact with phosphorylated serine and threonine residues (Muslin and Xing, 2000). Consistently, YAP phosphorylated on serine 127 interacts with 14-3-3 proteins (Zhao et al., 2007), which were also shown to mediate its association with $\alpha$-catenin (Schlegelmilch et al., 2011) in keratinocytes. To assess whether a similar complex could be detected in WT ECs, we immuno- precipitated YAP from EC extracts ( $36 \mathrm{~h}$ of culture). We were able to detect a VE-cadherin- $\alpha$-catenin-14-3-3-YAP complex from which EPS8 was excluded (Fig. 6 A). In contrast, $\alpha$-catenin and VE-cadherin, but neither YAP nor 14-3-3 proteins, were recovered in EPS8 immunoprecipitates. These results support the idea of two different, mutually exclusive pools of VE-cadherin. EPS8 may compete with the 14-3-3-YAP complex for the interaction with $\alpha$-catenin, ultimately controlling YAP activation by inhibiting its retention at AJs. We verified this hypothesis using recombinant purified proteins in in vitro pull-down 
A

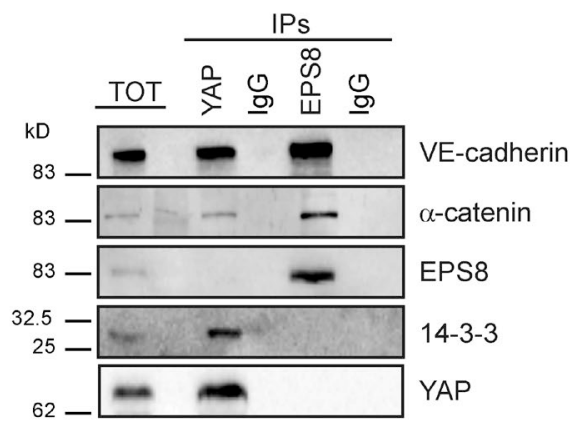

B
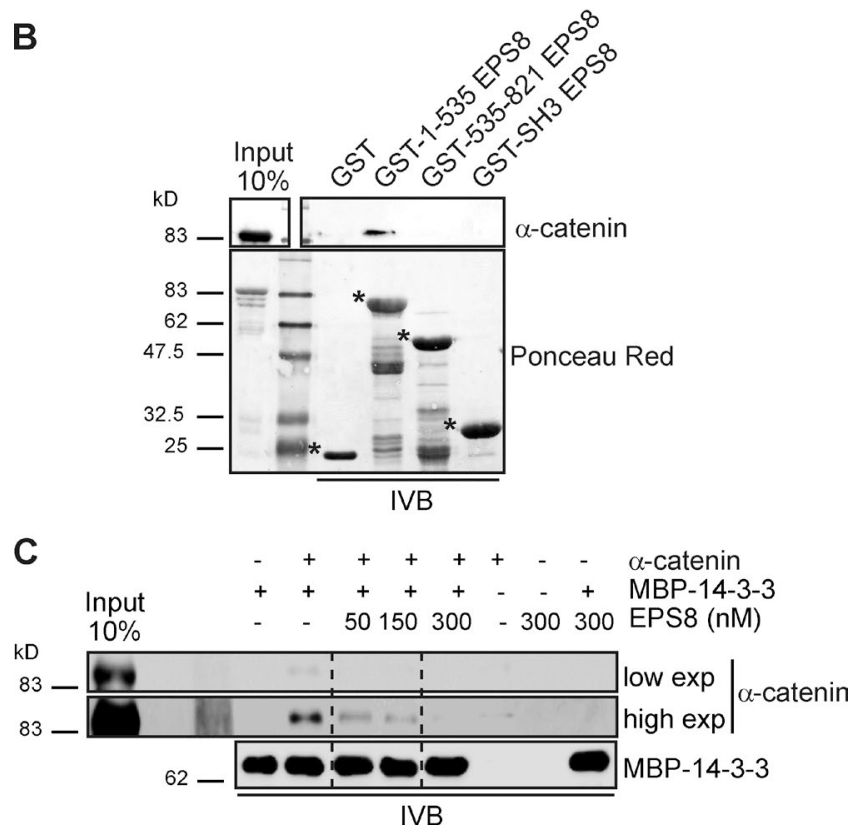

Figure 6. EPS8 and 14-3-3-YAP complex compete for binding to $\alpha$ catenin. (A) IP of YAP or EPS8 from VE-cadherin-positive cell extracts followed by WB analysis of $\alpha$-catenin, 14-3-3, and VE-cadherin association. EPS8 and YAP-14-3-3 are mutually exclusively bound to VE-cadherin and $\alpha$-catenin. (B) Analysis of the interaction between $\alpha$-catenin and EPS8deleted mutants (asterisks) in vitro by GST pull-down assay. (C) In vitro competition assay between EPS 8 and 14-3-3 for binding with $\alpha$-catenin. 14-3-3 bound $\alpha$-catenin in the absence of EPS8, and EPS8 reduced this direct interaction in a dose-dependent manner. The dashed lines indicate the blot has been cropped. IVB, in vitro binding; TOT, total cell lysate.

experiments. We found that EPS 8 directly bound $\alpha$-catenin through its N-terminal domain (Fig. 6 B). This latter observation suggested that EPS8 and the 14-3-3-YAP complex may compete for binding to $\alpha$-catenin. By monitoring the direct association of purified $\alpha$-catenin to immobilized 14-3-3 proteins (Schlegelmilch et al., 2011), we found that this interaction was abrogated in the presence of an equimolar amount of EPS8 (Fig. 6 C), supporting the idea of a competition between EPS8 and 14-3-3-YAP for $\alpha$-catenin binding.

A critical role of YAP is to release epithelial cells from contact inhibition of cell growth (Zhao et al., 2011). This latter phenomenon is induced by the formation of cadherin junctional complexes that, in addition to physically sequestering YAP at junctions (Zhao et al., 2011), also promote YAP phosphorylation, further impairing YAP nuclear translocation and transcriptional activity (Dupont et al., 2011). The growth inhibitory function was ascribed to VE-cadherin in ECs (Caveda et al., 1996; Giampietro et al., 2012), suggesting that EPS8 may contribute to this effect through the regulation of VE-cadherin stability at junctions (Fig. 2) and the consequent modulation of YAP localization and activity (Fig. 4).

To provide evidence in this direction, we monitored contact inhibition of cell growth in WT ECs, and we found that with the establishment of cell confluence, both Akt and YAP increased their phosphorylation level (Fig. 7 A), and in parallel YAP transcriptional activity was reduced (Fig. 7 B). Consistently, the removal of EPS8, which both dampens YAP activity and increases VE-cadherin localization at junctions, significantly reduced the number of confluent cells needed to achieve growth arrest (Fig. $7 \mathrm{C}$ ).

A correct control of proliferation and the proper establishment of junctional complexes are the crucial steps for the fine regulation of permeability exerted by ECs. Thus, we analyzed whether the absence of Eps8 and the consequent alteration in the transcriptional activity of YAP could impact the regulation of permeability. We found that Eps8 ${ }^{-}$ ECs displayed increased permeability compared with Eps $8^{+}$ ECs (Fig. 7 D). EPS8 is therefore emerging as a key inductor of YAP activity that acts both by preventing Akt-dependent phosphorylation of YAP, as well as by restraining the binding of the 14-3-3-YAP complex to $\alpha$-catenin, allowing YAP nuclear translocation.

These EPS8 activities are novel and unrelated to its well-established role in remodeling actin cytoskeleton. Indeed, ECs expressing an EPS8 mutant devoid of actin capping and bundling functions (Hertzog et al., 2010) showed a YAP activity almost equal to EPS8+-expressing cells as measured by serine 127 phosphorylation levels (Fig. 8 A) and by the induction of target gene expression (Fig. 8 B). So far, we have deciphered a new molecular mechanism (Fig. $8 \mathrm{C}$ ) through which EPS8, a novel partner of VE-cadherin, is able to modulate YAP transcriptional activity.

\section{The absence of EPSB alters YAP activity in vivo}

To confirm the in vivo relevance of the mechanisms described in the previous paragraphs, we analyzed YAP nuclear localization in vessels of WT and eps8-null mice. Consistent with what we previously observed, YAP nuclear localization was increased in the vasculature of eps8-null mice (Fig. 9, A and B). Furthermore, freshly isolated ECs obtained from eps8-null mice showed $\sim 30 \%$ reduction of YAP target gene expression compared with WT mice (Fig. 9 C). Finally, in agreement with cultured cells, the absence of eps 8 increased VE-cadherin localization at AJs in vivo too (Fig. $10 \mathrm{~A}$ ).

To test whether the observed defects in permeability shown in cultured ECs were also present in vivo, we tested small- and large-size tracers (cadaverine-Alexa Fluor 555 and $0.1-\mu \mathrm{m}$-diameter green fluorescent microsphere, respectively) in WT and eps8-null mice (Figs. $10 \mathrm{~B}$ and S5). The analysis revealed a specific increase $(\sim 30 \%)$ in the accumulation of cadaverine in the parenchyma of brain and lungs in eps8-null mice (Fig. 10 B), whereas no difference in the extravasation of the high-size green fluorescent microspheres was found (Fig. S5).

Collectively, these findings support the idea that the signaling mechanisms uncovered by in vitro analyses are present also in vivo, and Eps8 plays a role in controlling permeability, but only to small molecular size tracers. 


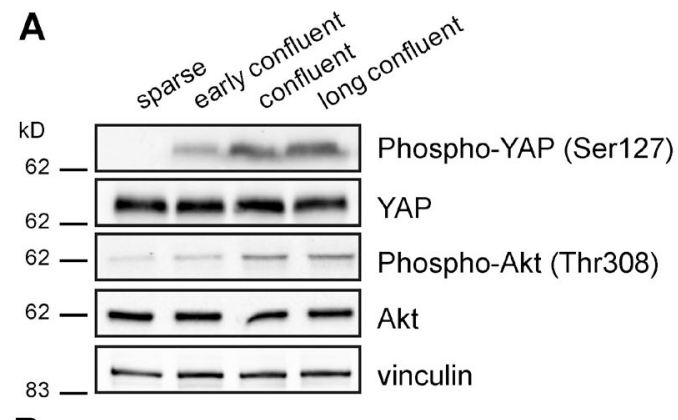

B

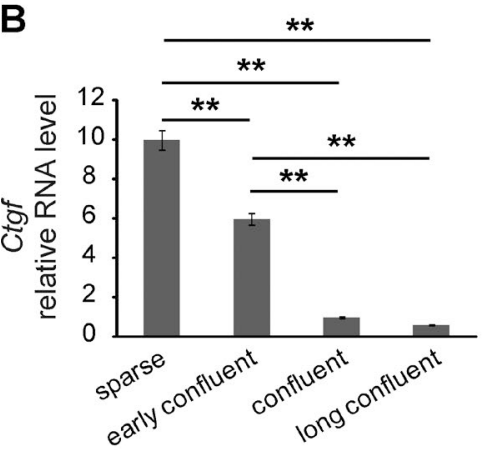

C

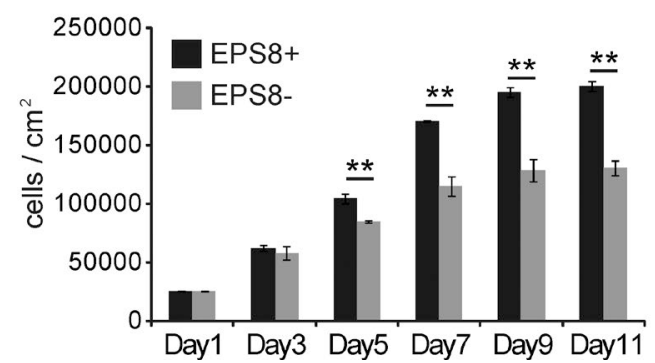

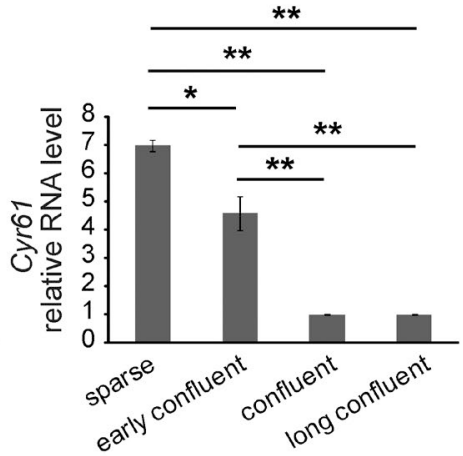

D
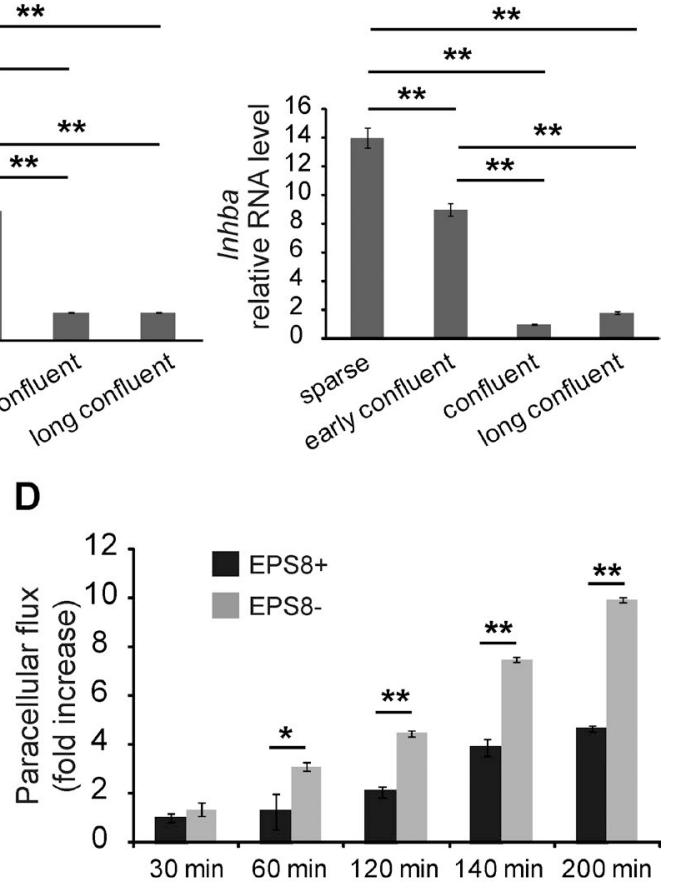

Figure 7. The absence of EPS8 induces earlier contact inhibition of cell growth and impairs EC permeability. (A) WB analysis of Phospho-YAP, YAP, Phospho-Akt, and Akt in WT lung-derived ECs at different stages of confluence conditions. Vinculin is the loading control. (B) qRT-PCR analysis of YAP target genes in WT lung-derived ECs at different stages of confluence conditions. Data are means \pm SEM of four independent experiments. (C) Cell density analysis performed on EPS8+ and EPS8- ECs. Equal numbers of cells were seeded at day 1 and subsequently counted at set time points. In the absence of EPS8, VE-cadherin localization is higher, and, consequently, cell-cell contact inhibition of proliferation is increased. Data are means \pm SEM of eight independent experiments. (D) Paracellular tracer flux assay. Permeability to FITC-dextran (70 kD) was assayed. Data are means \pm SEM of three independent experiments. At all time values from 60 min onward, Eps8+ ECs were statistically lower than Eps8- ECs. ${ }^{*}, \mathrm{P}<0.05 ;{ }^{* *}, \mathrm{P}<0.01$.

\section{Discussion}

Endothelial cell-to-cell junctions not only maintain intercellular adhesion but also transfer multiple intracellular signals that modulate contact inhibition of cell growth, cell polarity, lumen formation, and permeability (Dejana et al., 2009; McCrea et al., 2009). The complexity of VE-cadherin signaling is a result of the large number of identified intracellular partners that have been shown to be directly or indirectly associated with AJs in the endothelium (Dejana and Vestweber, 2013).

These signaling proteins can assemble into distinct types of complexes, which would vary in composition in the different vessels, stages of development, and even within the same cell. Furthermore, VE-cadherin association with one or another partner is reversible and can be spatially and temporally regulated.

We report here the identification of EPS8 as a new partner of the VE-cadherin complex in ECs that mediates the transduction of signals impinging on the regulation of the transcriptional coactivator YAP. EPS8 promotes VE-cadherin phosphorylation and ubiquitination. These posttranslational modifications in- variably associate with increased internalization and enhance cell surface turnover of VE-cadherin (Gavard and Gutkind, 2006; Orsenigo et al., 2012). Notably, EPS8 is not a stable component of VE-cadherin junctions, but it is transiently and rapidly recruited at cell-to-cell contacts during dynamic remodeling of junctions in early stages of confluency It is likely, therefore, that EPS8 contributes to increase junction dynamics. Conversely, EPS8 loss reduces the turnover of VE-cadherin and favors its clustering, a condition previously shown to promote the activation of the $\mathrm{PI}(3) \mathrm{K}$-Akt pathway (Carmeliet et al., 1999; Taddei et al., 2008). Consistent with our results, Choi and Kwon (2015) recently found that YAP subcellular localization and activity in ECs are regulated by the VE-cadherinmediated PI3K-Akt pathway. In keeping with these notions, we showed that the removal of EPS8 correlates with an increased localization of VE-cadherin at AJs and increased activation of the PI(3)K-Akt pathway.

YAP has long been shown to be a direct downstream target of Akt (Basu et al., 2003). This posttranslational modification creates binding sites for 14-3-3 proteins, a family of 
A

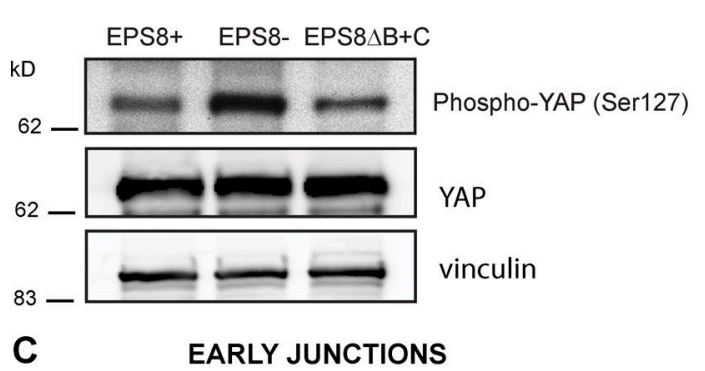

B

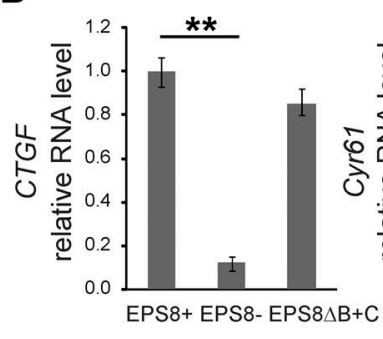

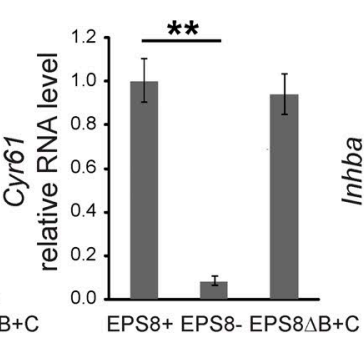

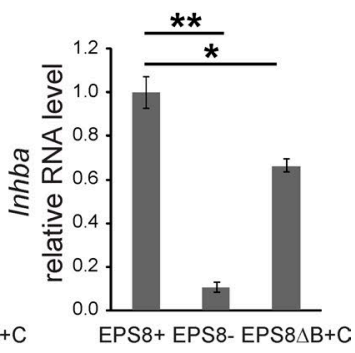

STABLE JUNCTIONS
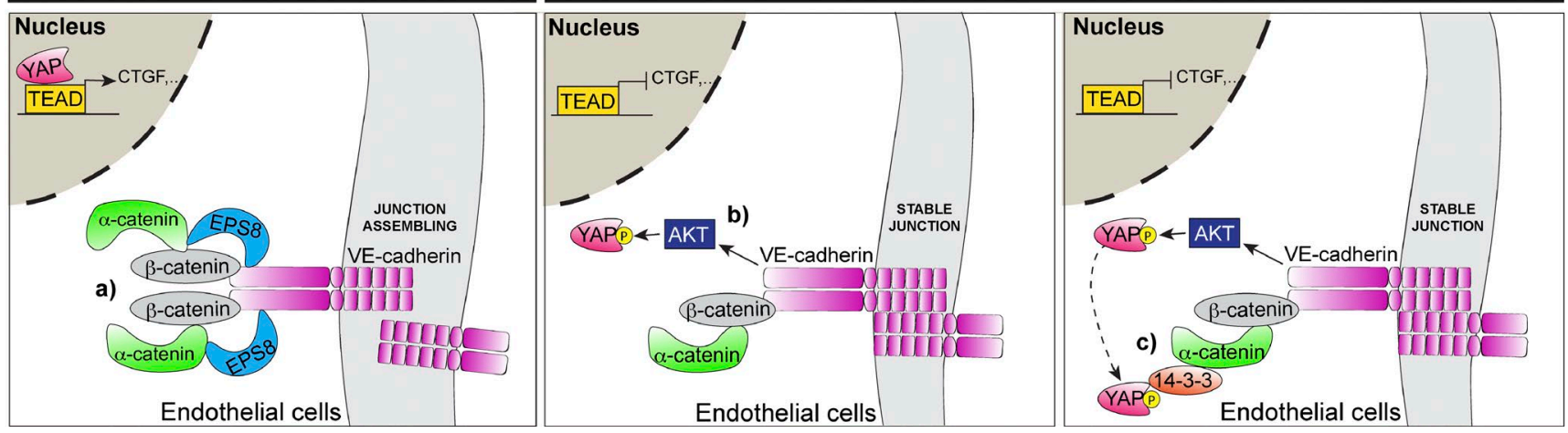

Figure 8. EPS8 modulation of YAP phosphorylation and transcriptional activity is independent from its actin capping and bundling functions. (A) WB analysis of YAP phosphorylation in EPS8+, EPS8-, and EPS8 $\triangle B+C$ ECs. Vinculin is shown as a loading control. (B) qRT-PCR analysis of Ctgf, Cyr61, and Inhba in confluent EPS8 ${ }^{+}$, EPS8- ${ }^{-}$, and EPS8 $\triangle B+C$ cells. Significant differences in YAP transcriptional activity has been detected only in EPS8- ECs. For each tested gene, the expression level has been represented as fold changes \pm SEM of three independent experiments. ${ }^{*}, P<0.05$; ${ }^{* *}, P<0.01$. (C) Suggested model for the regulation of YAP phosphorylation, localization, and transcriptional activity. EPS8 transiently binds VE-cadherin during junctional remodeling a) while YAP is localized in the nuclei of ECs; b) when junctions are stabilized, VE-cadherin clustering induces PI(3)K-Akt-YAP phosphorylation and, c) consequently, YAP is sequestered at the plasma membrane. a) The transient direct bond of Eps8 to $\alpha$-catenin prevents that of 14-3-3-phospho-YAP.

phosphoserine-binding proteins, that may retain YAP in the cytoplasm, preventing its nucleocytoplasmic trafficking and transcriptional activity (Zhao et al., 2007). By associating with 14-3-3, YAP was shown to bind $\alpha$-catenin in epithelial cells (Schlegelmilch et al., 2011). We report here that this interaction also occurs in ECs, where the loss of EPS8 promotes the localization of hyperphosphorylated YAP at AJs in a trimeric complex with 14-3-3 and $\alpha$-catenin.

We also found that EPS8 is able to bind directly to $\alpha$ catenin, competing with the 14-3-3-YAP complex. Coimmunoprecipitation experiments show the existence of two different, mutually exclusive protein complexes of VE-cadherin at AJs: in one complex, VE-cadherin binds $\alpha$-catenin and EPS8, whereas in the other, VE-cadherin binds $\alpha$-catenin, 14-3-3, and YAP. As a consequence, elevation of EPS8 levels inhibits, by competition for binding to $\alpha$-catenin, the formation of the YAP-14-3-3- $\alpha$-catenin protein assembly, ultimately inducing YAP nucleocytoplasmic shuttling and transcriptional activity. The exclusion of YAP from cell junctions by EPS8 is of particular relevance under conditions of dynamic remodeling of junctions, when endothelial monolayers may not have yet committed to a full growth arrest.

It was shown that actin cytoskeleton and tensional forces can modulate YAP activity (Dupont et al., 2011; Aragona et al., 2013). EPS8 is an actin-capping and -bundling protein that influences actin dynamics in migratory cells. This function resides in the C-terminal effector region of EPS8. We show here that EPS 8 can interact with $\alpha$-catenin through its $\mathrm{N}$-terminal domain at junctions in a topological arrangement that would enable EPS8 to execute its actin regulatory activity via its free effector C-terminal domain. However, the finding that an EPS8 mutant unable to interact with actin (Hertzog et al., 2010) is fully competent in restoring YAP translocation to the nucleus in EPS8-null ECs argues against this possibility. Our findings reveal, instead, an unexpected way of signaling of Eps8 that is apparently independent from its ability to control actin dynamics, but relies on a specific set of protein-protein interactions.

It will be important in future studies to determine the mechanism through which EPS8 is only transiently recruited to junctions. It is possible that increased tension across junctions is the key initiating cue that translates into the formation of a set of specialized complexes required to promote the necessary dynamics and plasticity of otherwise relatively stable structures and tissues.

Under in vitro and in vivo conditions, AJs are highly dynamic structures. Conditions that perturb this equilibrium might also perturb vascular permeability.

In eps8-null mice, VE-cadherin localization is increased at cell-to-cell contacts, likely through inhibition of turnover. As in cultured cells, eps8-null mice show an increase in vascular permeability, and the small-size tracer cadaverin accumulates in the parenchyma of different organs. However, large-size fluorescent beads did not cross the vessels more efficiently in the absence of eps8, suggesting a size-selective impairment of permeability. This result is consistent with the fact that eps8null mice are viable and fertile and apparently devoid of macroscopic vascular abnormalities.

A possible explanation for the absence of a more dramatic phenotype is that inactivation of Eps 8 may be compensated for by other members of the family of related genes (Scita et al., 1999). Double and triple knockout of EPS8-related genes may clarify this aspect. Alternatively, it is possible that under 

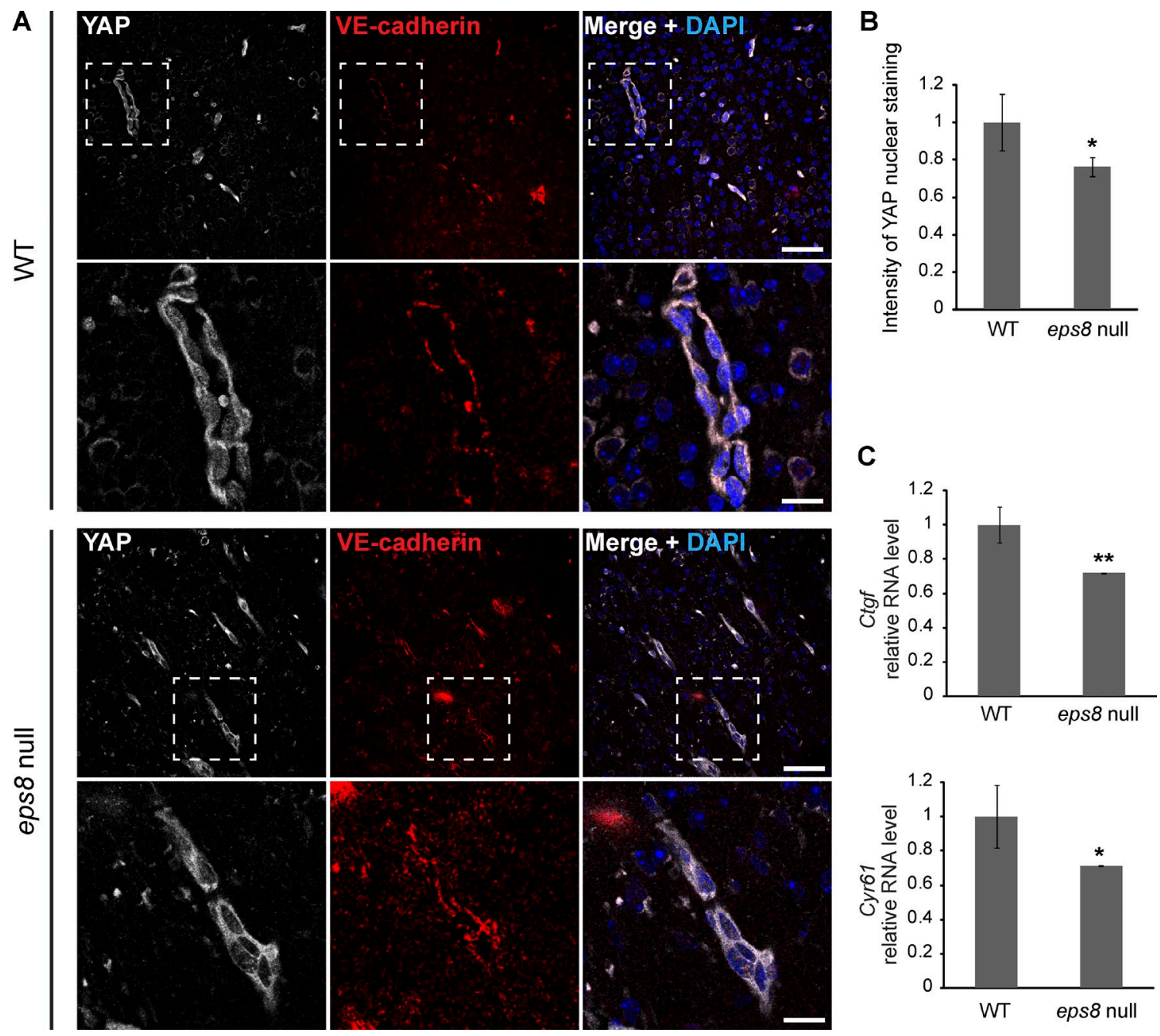

specific pathological conditions, eps8-null mice present alterations that are undetectable in healthy conditions.

In conclusion, we show here that the transient localization of EPS8 at endothelial junctions modulates VE-cadherin organization and induces YAP nuclear translocation and transcriptional activity. Further studies are required to characterize in more detail the relevance of this novel molecular pathway in additional in vivo models.

\section{Materials and methods}

\section{Cell lines}

Murine ECs genetically ablated for Cdh5 (VE-cadherin null) and modified to express the human WT VE-cadherin (VE-cadherin positive) or VE-cadherin $\Delta$-p120 (lacking aa 621-702 of human VE-cadherin cDNA, which correspond to the p120-cateninbinding region) and $\Delta$ - $\beta$ cat (lacking aa $703-784$ of human VEcadherin cDNA, which correspond to the $\beta$-catenin-binding region) were obtained and cultured as described previously (Giampietro et al., 2012). ECs isolated from lungs (Dong et al., 1997; Balconi et al., 2000) of eps8-null adult mice were lentivirally infected with EGFP alone or EGFP-EPS8 (Menna et al., 2009). For the experiments, 1,800 cells $/ \mathrm{cm}^{2}$ and 42,000 cells $/ \mathrm{cm}^{2}$ were seeded to obtain sparse and confluent cultures; and 40,000 cells/ $\mathrm{cm}^{2}$ were seeded and cultured for $24 \mathrm{~h}, 36 \mathrm{~h}$, and $72 \mathrm{~h}$ to reach different stages of confluency (early confluent, confluent, and long confluent, respectively). For all ECs of murine origin, the culture medium was DMEM with $20 \%$ FCS, 2-mM glutamine, 100 U/liter penicillin/streptomycin, 1-mM sodium pyruvate, $100 \mu \mathrm{g} / \mathrm{ml}$ heparin (from porcine intestinal mucosa; Sigma-Aldrich), and $5 \mu \mathrm{g} / \mathrm{ml}$ EC growth supplement (made from calf brain; complete culture medium).

The starving medium was MCDB 131 (Invitrogen) with 1\% BSA (EuroClone), 2-mM glutamine, $100 \mathrm{U} /$ liter penicillin/streptomycin, and $1-\mathrm{mM}$ sodium pyruvate.

The epithelial AD-HEK293 cell line (human embryonic kidney; American Type Culture Collection) used for adenoviral production was grown in DMEM (Cambrex Bioscience) supplemented with $10 \%$ FBS (HyClone), 2-mM glutamine, 100 U/liter penicillin/streptomycin, and 1-mM sodium pyruvate. 
A

5
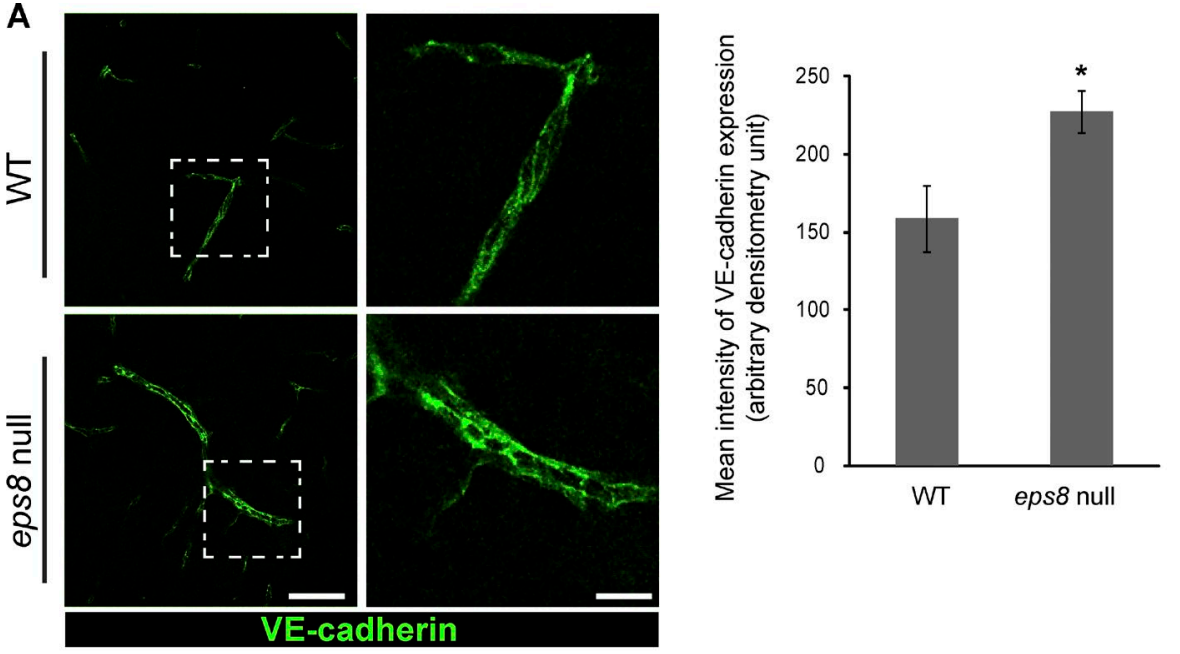

B

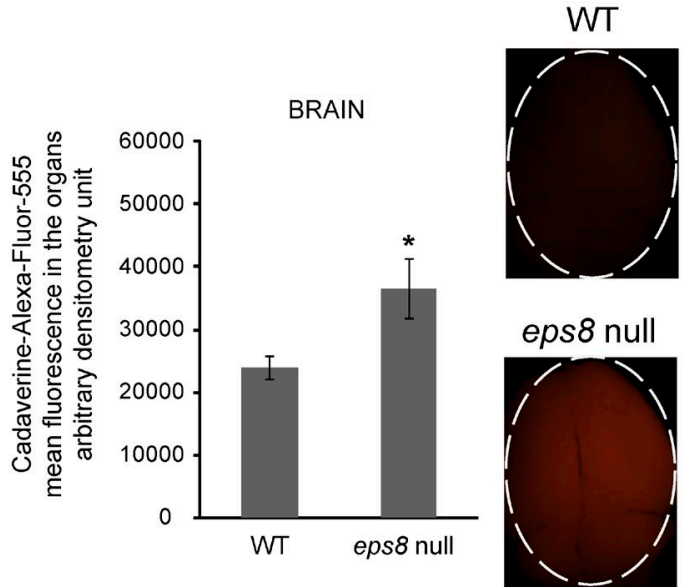

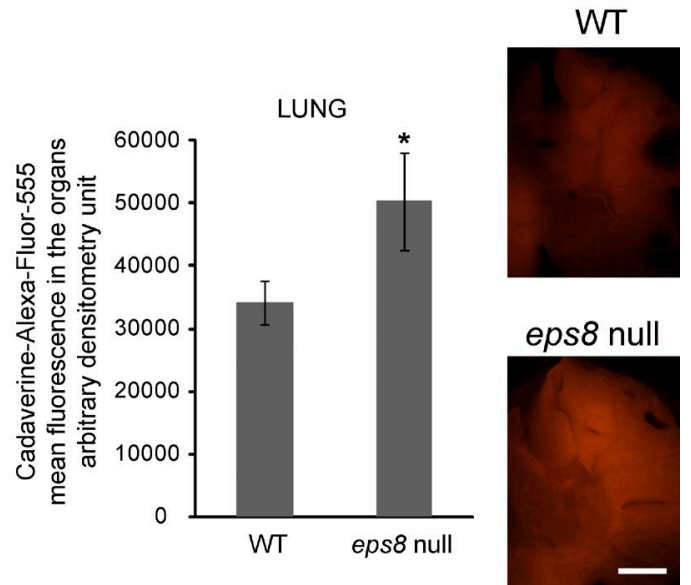

The COS-1 cell line (monkey kidney fibroblast-like cells; American Type Culture Collection) used for transient transfection was grown in DMEM supplemented with 10\% FBS and 2-mM glutamine. All cells were cultured at $37^{\circ} \mathrm{C}$ in a humidified atmosphere of $5 \% \mathrm{CO}_{2} /$ air.

\section{Antibodies}

For IF, Western blotting, and immunoprecipitation (IP), the following antibodies were used: VE-cadherin (C-19) goat (sc-6458; Santa Cruz Biotechnology, Inc.); VE-cadherin rat BV13 (ab91064; Abcam; Corada et al., 2002); EPS8 mouse (610144; BD); $\alpha$-tubulin mouse (T9026; Sigma-Aldrich); vinculin mouse (V9264; Sigma-Aldrich); Ub P4D1 mouse (sc-8017; Santa Cruz Biotechnology, Inc.); pY665VE-cadherin rabbit (gift from J. Gavard, Institut Cochin, Paris, France; Gavard and Gutkind, 2006); YAP (63.7) mouse (sc-10199; Santa Cruz Biotechnology, Inc.; Western blot [WB]); phospho-YAP (serine 127) rabbit (4911; Cell Signaling Technology); Rac mouse (610650; BD); YAP (H-9) mouse (sc-271134; Santa Cruz Biotechnology, Inc.; IF); pan 14-3-3 (K-19) rabbit (sc-629; Santa Cruz Biotechnology, Inc.); $\alpha$-catenin rabbit (C2081; Sigma-Aldrich; WB); $\alpha$-catenin rabbit (2028-1; Epitomics; IP); $\beta$-catenin mouse (610154; BD); Mst1 rabbit (3682; Cell Signaling Technology); Mst2 rabbit (3681; Cell Signaling
Technology); phospho-Mst1 (threonine 183)/Mst2 (threonine 180) rabbit (3681; Cell Signaling Technology); LATS1 (G-16) goat (sc12494; Santa Cruz Biotechnology, Inc.); phospho-LATS 1 (serine 909) rabbit (9157; Cell Signaling Technology); Akt rabbit (9271; Cell Signaling Technology); phospho-Akt (threonine 308) rabbit (9275; Cell Signaling Technology); Pecam-1 (CD31; ab28364; Abcam); HRPlinked anti-mouse, anti-rat, and anti-rabbit (Cell Signaling Technology); HRP-linked anti-goat (Promega); Alexa Fluor 555-conjugated donkey anti-mouse and anti-goat (Invitrogen); Alexa Fluor 488conjugated donkey anti-mouse (Invitrogen); and GST (Z-5) rabbit (sc-459; Santa Cruz Biotechnology, Inc.).

\section{Quantitative RT-PCR (qRT-PCR) analysis}

Total RNA was isolated using the RNeasy mini kit (QIAGEN), and $1 \mu \mathrm{g}$ was reverse transcribed with random hexamers (High Capacity cDNA Archive kit; Applied Biosystems). cDNA was amplified with the TaqMan Gene Expression assay (Applied Biosystems) and a thermocycler (ABI Prism 7900HT; Thermo Fisher Scientific). For any sample, the expression level, normalized to the housekeeping genes encoding $18 \mathrm{~S}$, was determined by the comparative threshold cycle method as described previously (Spagnuolo et al., 2004). 


\section{Mice}

All procedures involving animals and their care were performed in conformity with the guidelines established by the Italian Foundation for Cancer Research Institute of Molecular Oncology FoundationEuropean Institute of Oncology Campus Principles of Laboratory Animal Care (directive 86/609/EEC).

The generation of eps $8^{-1-}$ mice has been described previously by Scita et al. (1999). In brief, we isolated mouse genomic eps 8 clones from a 129SV library (Agilent Technologies). We used a 7-kb XhoIXhoI fragment for $5^{\prime}$ homology and a $2.5-\mathrm{kb}$ EcoRV-NotI fragment for $3^{\prime}$ homology. A phosphoglycerate kinase-neo cassette replaced an exon-containing eps 8 genomic $1.7-\mathrm{kb}$ XhoI-EcoRV fragment. The eps $8 \mathrm{SH} 3$ domain is encoded by two exons, and the targeting construct excluded the first and part of the second of these exons. A genomic probe, flanking the targeting construct at the $5^{\prime}$ end, was used to detect the WT $(2.6 \mathrm{~kb})$ and targeted $(9.5 \mathrm{~kb})$ alleles. Of note, this genetic lesion resulted in the complete loss of the eps 8 gene product as determined by mRNA analysis and immunoblotting with antipeptide serum raised against the N-terminal region of EPS8. Electroporation into mouse embryonic day 14 embryonic stem cell clones, and subsequent manipulations leading to mice heterozygous and homozygous for the mutant Eps 8 allele, were performed as described previously (Levéen et al., 1994). A targeted embryonic stem cell clone was injected into C57BL/6 blastocysts, and germline chimeras and mice heterozygous and homozygous for the eps 8 mutant allele were derived. DNA analyses, derivation of chimeras, and subsequent identification of germline transmission, and mice heterozygous and homozygous for the mutant eps8 allele, were described previously (Levéen et al., 1994). eps8-null mice were backcrossed for $>20$ generations to C57BL/6 mice. Age- and sex-matched C57BL/6 mice were used as controls.

\section{Intravenous injection of lysine-fixable cadaverine conjugated to Alexa Fluor $\mathbf{5 5 5}$ and microsphere}

Cadaverine conjugated to Alexa Fluor $555(3.125 \mathrm{mg} / \mathrm{ml}$ in saline) was injected intravenously into the tail vein of adult ( $2 \mathrm{mo}$ old) $25-\mathrm{mg} / \mathrm{kg}$ mice eps 8 null and controls. The circulation time was $2 \mathrm{~h}$. For in situ detection of cadaverine, the anesthetized mice were perfused for 1-2 min with HBSS, followed by $5 \mathrm{~min}$ of perfusion with 4\% PFA in PBS, pH 7.2. The organs were then removed and postfixed in $4 \%$ PFA at $4^{\circ} \mathrm{C}$ for $5-6 \mathrm{~h}$. Images of dissected organs were captured using a stereomicroscope (SZX16; Olympus) equipped with a fluorescence long-pass filter for RFP (excitation, 530-550 nm; emission, $575 \mathrm{~nm}$ ). Image acquisition was performed using a $1 \times$ objective with a total magnification of $0.35 \times$, supported by an RGB camera (Digital Sight DS-5Mc; Nikon). The ImageJ open-source software (National Institutes of Health) was used for data analysis. The mean fluorescence was calculated as the ratios of the total fluorescence signals to the number of pixels in the areas, expressed as arbitrary units.

For in situ detection of microspheres, the anesthetized mice were intravenously injected with green fluorescent microspheres (0.1$\mu \mathrm{m}$ diameter; $50 \mathrm{ml}$; Duke Scientific) and then perfused for 1-2 min with HBSS, followed by 5 min of perfusion with $4 \%$ PFA in PBS, pH 7.2. The tracheas were then removed and postfixed in $4 \%$ PFA at $4^{\circ} \mathrm{C}$ for $1 \mathrm{~h}$ and then processed for IF analyses.

\section{Histology and tissue IF}

Mouse organs were embedded in optimal cutting temperature compound (Tissue-Tek; Sakura) and snap frozen or embedded in paraffin. 5 - $\mu$ m-thick sections were cut. Frozen sections were fixed in cold methanol or 4\% PFA and subjected to IF. Paraffin sections were subjected to IF. Blocking ( $2 \mathrm{~h}$ ), primary (overnight), and secondary ( $3 \mathrm{~h}$ ) antibodies were diluted in PBS with 2\% BSA. Sections were then counterstained with DAPI and mounted in Vectashield.

\section{Retinal immunohistochemistry}

Eyes from WT C57BL/6 postnatal day 9 mice were fixed in 2\% PFA overnight before retinas were dissected. Retinas were incubated in 5\% donkey serum, $1 \%$ BSA, and $0.5 \%$ Triton X-100 in PBS overnight and the day after, stained with primary antibodies overnight. Then retinas were incubated with fluorophore-conjugated antibodies and mounted with ProLong gold (Invitrogen).

\section{IF microscopy}

Cells were cultured and then fixed with 4\% PFA or, if specified in the text, with $1 \%$ PFA in 2.5 -mM triethanolamine, $\mathrm{pH} 7.5$, containing $0.1 \%$ Triton X-100 and $0.1 \%$ NP-40 to optimize junctional staining. Fixed cells were permeabilized and incubated for $30 \mathrm{~min}$ in a blocking solution of PBS with $2 \%$ BSA.

Cells were then incubated overnight with primary antibodies diluted in blocking buffer. Appropriate secondary antibodies were applied on cells for $45 \mathrm{~min}$ at RT. Confocal microscopy was performed at RT with a confocal microscope (TCS SP2AOBS; Leica) equipped with violet (405-nm laser diode), blue (488 nm; Argon), yellow (561 nm; solid state), and red (633 nm; HeNe) excitation laser lines before processing with Photoshop (Adobe). Only adjustments of brightness and contrast were used in the preparation of the figures. For comparison purposes, different sample images of the same antigen were acquired under constant acquisition settings. Image acquisition was performed using a $63 \times / 1.4$ NA oil immersion objective (HCX PL APO 63× Lbd BL; Leica) with spectral detection bands and scanning modalities optimized for removal of channel cross talk. Confocal software (Leica) and ImageJ version 1.33 were used for data analysis.

\section{LUMIER assay}

The automated high throughput technology LUMIER to analyze dynamic protein-protein interaction networks in mammalian cells was performed as previously described (Barrios-Rodiles et al., 2005). Full-length human VE-cadherin and $\Delta$ - $\beta$ cat mutant were C-terminally tagged with RL (VE-cadherin-RL and $\Delta$ - $\beta$ cat-RL). In brief, HEK293T cells from a library of $6403 \times$ Flag-tagged cDNAs that encode proteins comprised of diverse signaling-associated domains (Miller et al., 2009), plated in dishes, were robotically transfected using PolyFect (QIAGEN). After 48 h, cells were lysed and then immunoprecipitated using anti-Flag M2 monoclonal antibody (Sigma-Aldrich). Luciferase activity in immunoprecipitates and in aliquots of total cell lysates was determined using the Renilla Luciferase Assay system (Promega).

\section{Lung EC isolation}

Lungs were excised from mice and digested with collagenase type I (Roche) for $2 \mathrm{~h}$ at $37^{\circ} \mathrm{C}$. The ECs were then separated using Dynabeads (Invitrogen) coated with Pecam-1 antibody (BD) according to the manual's instructions and immediately processed for RNA isolation. cDNA synthesis and qRT-PCR were performed as described in the Quantitative RT-PCR (qRT-PCR) analysis section.

\section{Statistical analysis}

A Student's two-tailed unpaired $t$ test was used to determine statistical significance. The significance level was set at $\mathrm{P}<0.05$.

\section{IP}

Cells were incubated with $100 \mu \mathrm{g} / \mathrm{ml}$ dithiobis(succinimidyl)propionate (Thermo Fisher Scientific) for $20 \mathrm{~min}$ at $37^{\circ} \mathrm{C}$ and then solubilized in lysis buffer (100-mM Tris-HCl, $\mathrm{pH} 7.4,150-\mathrm{mM} \mathrm{NaCl}, 1 \%$ Triton $\mathrm{X}-100,1 \%$ deoxycholic acid, $0.1 \% \mathrm{SDS}, 2-\mathrm{mM} \mathrm{CaCl}_{2}$, and 
protease/phosphatase inhibitors) on ice for $30 \mathrm{~min}$. Precleared cell extracts were subjected to antibody precipitation overnight at $4{ }^{\circ} \mathrm{C}$, and immune complexes were captured by protein $\mathrm{G}-$ Sepharose beads (GE Healthcare). Immunoprecipitated material was separated on Trisglycine SDS-PAGE, blotted onto nitrocellulose membrane, and analyzed by standard methodologies.

\section{Western blotting}

Confluent cells were lysed by boiling in a modified Laemmli sample buffer (2\% SDS, $20 \%$ glycerol, and $125-\mathrm{mM}$ Tris-HCl, $\mathrm{pH} 6.8$ ). Equal amounts of proteins were loaded on gels, separated by SDSPAGE, and transferred to a nitrocellulose membrane (Protran; Whatman). After incubation with primary and HRP-linked secondary antibodies, specific bindings were detected by a chemiluminescence system (GE Healthcare).

\section{Gelatin-glutaraldehyde cross-linking}

To enhance EC adhesion, slides were coated with glutaraldehydecross-linked gelatin as follows. The culture supports were incubated for $1 \mathrm{~h}$ at RT with $1 \%$ gelatin, followed by a cross-linking with $2 \%$ glutaraldehyde solution for 15 min at RT. The glutaraldehyde was replaced by $70 \%$ ethanol. After $1 \mathrm{~h}$, five washes with PBS followed by overnight incubation with PBS containing 2-mM glycine were performed. Before cell seeding, slides were washed five times with PBS.

\section{Paracellular tracer flux analysis}

Cells were seeded on 6.5-mm-diameter Transwell permeable supports (pore size $0.4 \mu \mathrm{m}$; Corning), cultured in complete culture medium, and assayed for permeability to FITC-dextran (70 kD; Sigma-Aldrich). Next, FITC-dextran was added to the medium of the Transwell apical compartment at a concentration of $1 \mathrm{mg} / \mathrm{ml}$. At different times of incubation, a 50- $\mu \mathrm{l}$ aliquot of the medium was collected from the basal compartment, and the paracellular tracer flux was measured as the amount of FITC-dextran in the medium using a fluorometer (Wallac Victor3 1420 multilabel counter; PerkinElmer).

\section{Active Rho and Rac pull-down assay}

To detect active Rho, we used the Active Rho Pull-Down and Detection kit (Thermo Fisher Scientific) in accordance with the manufacturer's instructions. To detect active Rac, we used the G-LISA Rac activation assay (Cytoskeleton, Inc.) in accordance with the manufacturer's instructions.

\section{Treatments}

ECs were starved overnight before any treatment. 10- $\mu$ M LY294002 (Cell Signaling Technology) was added overnight at $37^{\circ} \mathrm{C}$.

\section{Constructs}

A form of Akt that is constitutively active (myr-Akt) was a gift from C. Daly (Regeneron Pharmaceuticals Inc., Tarrytown, NY). Cytomegalovirus promoter-based, elongation factor-1 promoter-based eukaryotic expression vectors, and GST bacterial expression vectors were generated by recombinant PCR. His6-tagged $\alpha$-catenin full-length was a gift from B. Weis and W.J. Nelson (Stanford University, Stanford, CA). MBP-14-3-3 was procured from GeneCopoeia. All constructs were verified by sequencing.

\section{Protein purification}

Recombinant full-length His-EPS8, His-Irsp53, and GST-EPS8 fragments were expressed and purified as previously described (Disanza et al., 2006, 2013; Hertzog et al., 2010). In brief, recombinant fragments were expressed as His- or GST-fusion proteins in the BL21 Escherichia coli strain (Agilent Technologies) and affinity purified using GS4B glutathione-Sepharose beads (GE Healthcare) or nickel-nitrilotriacetic acid agarose. Eluted proteins were dialyzed in 50-mM Tris- $\mathrm{HCl}, 150-\mathrm{mM} \mathrm{NaCl}, 1-\mathrm{mM}$ DTT, and $20 \%$ glycerol. GST-VE-cadherin intracellular domain was expressed in BL21 Rosetta strain (Agilent Technologies) and affinity purified using GS4B glutathione-Sepharose beads. His- $\alpha$-catenin was expressed in the BL21 E. coli strain (Agilent Technologies) and affinity purified using nickel-nitrilotriacetic acid (Ni-NTA) agarose according to standard procedures. Recombinant purified protein was eluted with $200-\mathrm{mM}$ imidazole and dialyzed in 50-mM Tris, pH 7.8, 150-mM NaCl, 1-mM DTT, and 5\% glycerol. MBP-14-3-3 was purified by standard procedures using amyloseSepharose affinity purification.

\section{In vitro binding assay}

MBP-14-3-3- $\alpha$-catenin \pm EPS8. Recombinant purified proteins were incubated overnight at $4^{\circ} \mathrm{C}$ in $\mathrm{Xb}$ buffer (50-mM Tris- $\mathrm{HCl}, \mathrm{pH} 7.4$, 150-mM NaCl, 0.02\% Triton X-100, 20-mM imidazole, 1-mM DTT, and protease inhibitor cocktail). Samples were then incubated for $1 \mathrm{~h}$ at $4{ }^{\circ} \mathrm{C}$ with amylose-Sepharose beads and washed three times with $\mathrm{Xb}$ buffer. Amylose-Sepharose beads were resuspended in a 1:1 volume of $2 \times$ SDS-PAGE sample buffer, boiled for $5 \mathrm{~min}$ at $95^{\circ} \mathrm{C}$, spun down for $1 \mathrm{~min}$, and loaded on acrylamide gels.

EPS8 full-length/VE-cadherin cytoplasmic tail. Recombinant purified His-EPS8 and VE-cadherin-C-terminal fragment were incubated for $1 \mathrm{~h}$ at $4^{\circ} \mathrm{C}$ with Ni-NTA beads in Xa buffer (50-mM Tris, $\mathrm{pH} 8$, 300-mM NaCl, 0.1\% Triton X-100, 20-mM imidazole, 1-mM DTT, and protease inhibitor cocktail). Samples were washed three times in $\mathrm{Xb}$ buffer. Beads were resuspended in a 1:1 volume of $2 \times$ SDS-PAGE sample buffer, boiled for $5 \mathrm{~min}$ at $95^{\circ} \mathrm{C}$, spun down for $1 \mathrm{~min}$, and loaded on acrylamide gels.

EPS8 fragments/ $\alpha$-catenin full length. Recombinant purified GST-EPS 8 fragments and His- $\alpha$-catenin were incubated for $1 \mathrm{~h}$ at $4{ }^{\circ} \mathrm{C}$ with Ni-NTA beads in Xb buffer. Samples were washed three times in $\mathrm{Xb}$ buffer. Beads were resuspended in a 1:1 volume of $2 \times$ SDS-PAGE sample buffer, boiled for $5 \mathrm{~min}$ at $95^{\circ} \mathrm{C}$, spun down for $1 \mathrm{~min}$, and loaded on acrylamide gels.

EPS8 fragments/VE-cadherin cytoplasmic tail. Equal amounts of cell lysates overexpressing VE-cadherin cDNA were incubated with GST-EPS8 fragments (GST as control) for $2 \mathrm{~h}$ at $4^{\circ} \mathrm{C}$ in the presence of GS4B glutathione-Sepharose beads. Samples were washed three times in lysis buffer. Beads were resuspended in a 1:1 volume of $2 \times$ SDSPAGE sample buffer, boiled for $5 \mathrm{~min}$ at $95^{\circ} \mathrm{C}$, spun down for $1 \mathrm{~min}$, and loaded on acrylamide gels.

\section{RNAi}

To interfere with $\alpha$-catenin, we used siRNA (ON-TARGETplus L-048960-01; SMARTpool duplex Ctnna1) from GE Healthcare and the corresponding nontargeting pool (ONTARGETplus). Transfection was performed with Lipofectamine 2000 (Invitrogen) in accordance with the manufacturer's instructions.

\section{Online supplemental material}

Fig. S1 describes EPS8 reconstitution in EPS8- ECs. Fig. S2 shows that YAP transcriptional activity is regulated by VE-cadherin. Fig. S3 shows that EPS8 expression does not influence the Hippo pathway and Rho and Rac activity in ECs. Fig. S4 shows that YAP binding to VE-cadherin negatively regulates its transcriptional activity. Fig. S5 shows the high-size permeability control in vivo. Online supplemental material is available at http://www.jcb.org/cgi/content/ full/jcb.201501089/DC1. 


\section{Acknowledgments}

We thank F. Orsenigo, F. Pisati, and L. Terreran for technical help.

This work was supported by grants from Associazione Italiana per la Ricerca sul Cancro (AIRC; 10168 to G. Scita and 14471 to E. Dejana), "Special Program Molecular Clinical Oncology 5x1000" (to AIRC-Gruppo Italiano Malattie Mieloproliferative), the European Research Council (projects EU-ERC 268870 to E. Dejana and EU-ERC 268836 to G. Scita), the European Community (ITN VESSEL 317250 , BtBRAIN to E. Dejana), the Telethon Foundation (GGP14149 to E. Dejana), the Italian Ministry of Education, University and Research (MIUR-PRIN-2009X23L78), the Italian Ministry of Health, the Association for International Cancer Research (09-0582 to G. Scita), the CAR IPLO Foundation (2010-0737 to G. Scita, 2011 1-0596 to A. Disanza, and 2012-0678 to E. Dejana), and the German Research Foundation (SFB829 to C. Niessen).

The authors declare no competing financial interests.

Submitted: 21 January 2015

Accepted: 10 November 2015

\section{References}

Aragona, M., T. Panciera, A. Manfrin, S. Giulitti, F. Michielin, N. Elvassore, S. Dupont, and S. Piccolo. 2013. A mechanical checkpoint controls multicellular growth through YAP/TAZ regulation by actin-processing factors. Cell. 154:1047-1059. http://dx.doi.org/10.1016/j.cell.2013.07.042

Auciello, G., D.L. Cunningham, T. Tatar, J.K. Heath, and J.Z. Rappoport. 2013. Regulation of fibroblast growth factor receptor signalling and trafficking by Src and Eps8. J. Cell Sci. 126:613-624. http://dx.doi.org/10.1242/jcs.116228

Balconi, G., R. Spagnuolo, and E. Dejana. 2000. Development of endothelial cell lines from embryonic stem cells: A tool for studying genetically manipulated endothelial cells in vitro. Arterioscler. Thromb. Vasc. Biol. 20:1443-1451. http://dx.doi.org/10.1161/01.ATV.20.6.1443

Barrios-Rodiles, M., K.R. Brown, B. Ozdamar, R. Bose, Z. Liu, R.S. Donovan, F. Shinjo, Y. Liu, J. Dembowy, I.W. Taylor, et al. 2005. High-throughput mapping of a dynamic signaling network in mammalian cells. Science. 307:1621-1625. http://dx.doi.org/10.1126/science.1105776

Basu, S., N.F. Totty, M.S. Irwin, M. Sudol, and J. Downward. 2003. Akt phosphorylates the Yes-associated protein, YAP, to induce interaction with 14-3-3 and attenuation of p73-mediated apoptosis. Mol. Cell. 11:1123. http://dx.doi.org/10.1016/S1097-2765(02)00776-1

Brown, D.J., E.M. Rzucidlo, B.L. Merenick, R.J. Wagner, K.A. Martin, and R.J. Powell. 2005. Endothelial cell activation of the smooth muscle cell phosphoinositide 3-kinase/Akt pathway promotes differentiation. J. Vasc. Surg. 41:509-516. http://dx.doi.org/10.1016/j.jvs.2004.12.024

Carmeliet, P., M.G. Lampugnani, L. Moons, F. Breviario, V. Compernolle, F. Bono, G. Balconi, R. Spagnuolo, B. Oosthuyse, M. Dewerchin, et al. 1999. Targeted deficiency or cytosolic truncation of the VE-cadherin gene in mice impairs VEGF-mediated endothelial survival and angiogenesis. Cell. 98:147-157. http://dx.doi.org/10.1016/S0092-8674(00)81010-7

Caveda, L., I. Martin-Padura, P. Navarro, F. Breviario, M. Corada, D. Gulino, M.G. Lampugnani, and E. Dejana. 1996. Inhibition of cultured cell growth by vascular endothelial cadherin (cadherin-5/VE-cadherin). J. Clin. Invest. 98:886-893. http://dx.doi.org/10.1172/JCI118870

Choi, H.J., H. Zhang, H. Park, K.S. Choi, H.W. Lee, V. Agrawal, Y.M. Kim, and Y.G. Kwon. 2015. Yes-associated protein regulates endothelial cell contact-mediated expression of angiopoietin-2. Nat. Commun. 6:6943. http://dx.doi.org/10.1038/ncomms7943

Corada, M., L. Zanetta, F. Orsenigo, F. Breviario, M.G. Lampugnani, S. Bernasconi, F. Liao, D.J. Hicklin, P. Bohlen, and E. Dejana. 2002. A monoclonal antibody to vascular endothelial-cadherin inhibits tumor angiogenesis without side effects on endothelial permeability. Blood. 100:905-911. http://dx.doi.org/10.1182/blood.V100.3.905

Croce, A., G. Cassata, A. Disanza, M.C. Gagliani, C. Tacchetti, M.G. Malabarba, M.F. Carlier, G. Scita, R. Baumeister, and P.P. Di Fiore. 2004. A novel actin barbed-end-capping activity in EPS-8 regulates apical morphogenesis in intestinal cells of Caenorhabditis elegans. Nat. Cell Biol. 6:1173-1179. http://dx.doi.org/10.1038/ncb1198
Dejana, E., and C. Giampietro. 2012. Vascular endothelial-cadherin and vascular stability. Curr. Opin. Hematol. 19:218-223. http://dx.doi.org/10.1097/ MOH.0b013e3283523e1c

Dejana, E., and D. Vestweber. 2013. The role of VE-cadherin in vascular morphogenesis and permeability control. Prog. Mol. Biol. Transl. Sci. 116:119-144. http://dx.doi.org/10.1016/B978-0-12-394311-8.00006-6

Dejana, E., E. Tournier-Lasserve, and B.M. Weinstein. 2009. The control of vascular integrity by endothelial cell junctions: Molecular basis and pathological implications. Dev. Cell. 16:209-221. http://dx.doi.org/10 1016/j.devcel.2009.01.004

Di Fiore, P.P., and G. Scita. 2002. Eps8 in the midst of GTPases. Int. J. Biochem. Cell Biol. 34:1178-1183. http://dx.doi.org/10.1016/S1357 $-2725(02) 00064-X$

Disanza, A., M.F. Carlier, T.E. Stradal, D. Didry, E. Frittoli, S. Confalonieri, A. Croce, J. Wehland, P.P. Di Fiore, and G. Scita. 2004. Eps8 controls actin-based motility by capping the barbed ends of actin filaments. Nat. Cell Biol. 6:1180-1188. http://dx.doi.org/10.1038/ncb1199

Disanza, A., S. Mantoani, M. Hertzog, S. Gerboth, E. Frittoli, A. Steffen, K. Berhoerster, H.J. Kreienkamp, F. Milanesi, P.P. Di Fiore, et al. 2006. Regulation of cell shape by $\mathrm{Cdc} 42$ is mediated by the synergic actinbundling activity of the Eps8-IRSp53 complex. Nat. Cell Biol. 8:13371347. http://dx.doi.org/10.1038/ncb1502

Disanza, A., S. Bisi, M. Winterhoff, F. Milanesi, D.S. Ushakov, D. Kast, P. Marighetti, G. Romet-Lemonne, H.M. Müller, W. Nickel, et al. 2013. CDC42 switches IRSp53 from inhibition of actin growth to elongation by clustering of VASP. EMBO J. 32:2735-2750. http://dx.doi.org/10.1038/ emboj.2013.208

Dong, Q.G., S. Bernasconi, S. Lostaglio, R.W. De Calmanovici, I. MartinPadura, F. Breviario, C. Garlanda, S. Ramponi, A. Mantovani, and A. Vecchi. 1997. A general strategy for isolation of endothelial cells from murine tissues. Characterization of two endothelial cell lines from the murine lung and subcutaneous sponge implants. Arterioscler. Thromb. Vasc. Biol. 17:1599-1604. http://dx.doi.org/10.1161/01.ATV.17.8.1599

Dupont, S., L. Morsut, M. Aragona, E. Enzo, S. Giulitti, M. Cordenonsi, F. Zanconato, J. Le Digabel, M. Forcato, S. Bicciato, et al. 2011. Role of YAP/TAZ in mechanotransduction. Nature. 474:179-183. http://dx.doi .org/10.1038/nature 10137

Frittoli, E., G. Matteoli, A. Palamidessi, E. Mazzini, L. Maddaluno, A. Disanza, C. Yang, T. Svitkina, M. Rescigno, and G. Scita. 2011. The signaling adaptor Eps8 is an essential actin capping protein for dendritic cell migration. Immunity. 35:388-399. http://dx.doi.org/10.1016/j.immuni.2011.07.007

Gavard, J., and J.S. Gutkind. 2006. VEGF controls endothelial-cell permeability by promoting the $\beta$-arrestin-dependent endocytosis of VE-cadherin. Nat. Cell Biol. 8:1223-1234. http://dx.doi.org/10.1038/ncb1486

Giampietro, C., A. Taddei, M. Corada, G.M. Sarra-Ferraris, M. Alcalay, U. Cavallaro, F. Orsenigo, M.G. Lampugnani, and E. Dejana. 2012. Overlapping and divergent signaling pathways of N-cadherin and VEcadherin in endothelial cells. Blood. 119:2159-2170. http://dx.doi.org/10 .1182/blood-2011-09-381012

Giannotta, M., M. Trani, and E. Dejana. 2013. VE-cadherin and endothelial adherens junctions: Active guardians of vascular integrity. Dev. Cell. 26:441-454. http://dx.doi.org/10.1016/j.devcel.2013.08.020

Goddard, L.M., and M.L. Iruela-Arispe. 2013. Cellular and molecular regulation of vascular permeability. Thromb. Haemost. 109:407-415. http://dx.doi .org/10.1160/TH12-09-0678

Hertzog, M., F. Milanesi, L. Hazelwood, A. Disanza, H. Liu, E. Perlade, M.G. Malabarba, S. Pasqualato, A. Maiolica, S. Confalonieri, et al. 2010. Molecular basis for the dual function of Eps8 on actin dynamics: Bundling and capping. PLoS Biol. 8:e1000387. http://dx.doi.org/10.1371 /journal.pbio.1000387

Kim, N.G., E. Koh, X. Chen, and B.M. Gumbiner. 2011. E-cadherin mediates contact inhibition of proliferation through Hippo signaling-pathway components. Proc. Natl. Acad. Sci. USA. 108:11930-11935. http://dx.doi org/10.1073/pnas.1103345108

Kluger, M.S., P.R. Clark, G. Tellides, V. Gerke, and J.S. Pober. 2013. Claudin-5 controls intercellular barriers of human dermal microvascular but not human umbilical vein endothelial cells. Arterioscler. Thromb. Vasc. Biol. 33:489-500. http://dx.doi.org/10.1161/ATVBAHA.112.300893

Lampugnani, M.G., M. Corada, P. Andriopoulou, S. Esser, W. Risau, and E. Dejana. 1997. Cell confluence regulates tyrosine phosphorylation of adherens junction components in endothelial cells. J. Cell Sci. 110:2065-2077.

Lanzetti, L., V. Rybin, M.G. Malabarba, S. Christoforidis, G. Scita, M. Zerial, and P.P. Di Fiore. 2000. The Eps8 protein coordinates EGF receptor signalling through Rac and trafficking through Rab5. Nature. 408:374377. http://dx.doi.org/10.1038/35042605

Levéen, P., M. Pekny, S. Gebre-Medhin, B. Swolin, E. Larsson, and C. Betsholtz. 1994. Mice deficient for PDGF B show renal, cardiovascular, and 
hematological abnormalities. Genes Dev. 8:1875-1887. http://dx.doi.org /10.1101/gad.8.16.1875

Manor, U., A. Disanza, M. Grati, L. Andrade, H. Lin, P.P. Di Fiore, G. Scita, and B. Kachar. 2011. Regulation of stereocilia length by myosin XVa and whirlin depends on the actin-regulatory protein Eps8. Curr. Biol. 21:167-172. http://dx.doi.org/10.1016/j.cub.2010.12.046

McCrea, P.D., D. Gu, and M.S. Balda. 2009. Junctional music that the nucleus hears: Cell-cell contact signaling and the modulation of gene activity. Cold Spring Harb. Perspect. Biol. 1:a002923. http://dx.doi.org/10.1101 /cshperspect.a002923

Menna, E., A. Disanza, C. Cagnoli, U. Schenk, G. Gelsomino, E. Frittoli, M. Hertzog, N. Offenhauser, C. Sawallisch, H.J. Kreienkamp, et al. 2009. Eps8 regulates axonal filopodia in hippocampal neurons in response to brain-derived neurotrophic factor (BDNF). PLoS Biol. 7:e1000138. http://dx.doi.org/10.1371/journal.pbio.1000138

Miller, B.W., G. Lau, C. Grouios, E. Mollica, M. Barrios-Rodiles, Y. Liu, A. Datti, Q. Morris, J.L. Wrana, and L. Attisano. 2009. Application of an integrated physical and functional screening approach to identify inhibitors of the Wnt pathway. Mol. Syst. Biol. 5:315. http://dx.doi.org /10.1038/msb.2009.72

Morgan, J.T., C.J. Murphy, and P. Russell. 2013. What do mechanotransduction, Hippo, Wnt, and TGF $\beta$ have in common? YAP and TAZ as key orchestrating molecules in ocular health and disease. Exp. Eye Res. 115:1-12. http://dx.doi.org/10.1016/j.exer.2013.06.012

Morin-Kensicki, E.M., B.N. Boone, M. Howell, J.R. Stonebraker, J. Teed, J.G. Alb, T.R. Magnuson, W. O’Neal, and S.L. Milgram. 2006. Defects in yolk sac vasculogenesis, chorioallantoic fusion, and embryonic axis elongation in mice with targeted disruption of Yap65. Mol. Cell. Biol. 26:77-87. http://dx.doi.org/10.1128/MCB.26.1.77-87.2006

Muslin, A.J., and H. Xing. 2000. 14-3-3 proteins: Regulation of subcellular localization by molecular interference. Cell. Signal. 12:703-709. http:// dx.doi.org/10.1016/S0898-6568(00)00131-5

Navarro, P., L. Caveda, F. Breviario, I. Mândoteanu, M.G. Lampugnani, and E. Dejana. 1995. Catenin-dependent and -independent functions of vascular endothelial cadherin. J. Biol. Chem. 270:30965-30972. http://dx .doi.org/10.1074/jbc.270.52.30965

Orsenigo, F., C. Giampietro, A. Ferrari, M. Corada, A. Galaup, S. Sigismund, G. Ristagno, L. Maddaluno, G.Y Koh, D. Franco, et al, 2012. Phosphorylation of VE-cadherin is modulated by haemodynamic forces and contributes to the regulation of vascular permeability in vivo. Nat. Commun. 3:1208. http://dx.doi.org/10.1038/ncomms2199

Pannekoek, W.J., J.J. van Dijk, O.Y. Chan, S. Huveneers, J.R. Linnemann, E. Spanjaard, P.M. Brouwer, A.J. van der Meer, F.J. Zwartkruis, H. Rehmann, et al. 2011. Epac1 and PDZ-GEF cooperate in Rap1 mediated endothelial junction control. Cell. Signal. 23:2056-2064. http://dx.doi.org/10.1016/j.cellsig.2011.07.022

Ribatti, D., B. Nico, A. Vacca, L. Roncali, and F. Dammacco. 2002. Endothelial cell heterogeneity and organ specificity. J. Hematother. Stem Cell Res. 11:81-90. http://dx.doi.org/10.1089/152581602753448559

Schlegelmilch, K., M. Mohseni, O. Kirak, J. Pruszak, J.R. Rodriguez, D. Zhou, B.T. Kreger, V. Vasioukhin, J. Avruch, T.R. Brummelkamp, and
FD Camargo 2011. Yap1 acts downstream of $\alpha$-catenin to control epidermal proliferation. Cell. 144:782-795. http://dx.doi.org/10.1016 /j.cell.2011.02.031

Scita, G., J. Nordstrom, R. Carbone, P. Tenca, G. Giardina, S. Gutkind, M. Bjarnegård, C. Betsholtz, and P.P. Di Fiore 1999. EPS8 and E3B transduce signals from Ras to Rac. Nature. 401:290-293. http://dx.doi org/10.1038/45822

Silvis, M.R., B.T. Kreger, W.H. Lien, O. Klezovitch, G.M. Rudakova, F.D. Camargo, D.M. Lantz, J.T. Seykora, and V. Vasioukhin. 2011. $\alpha$-catenin is a tumor suppressor that controls cell accumulation by regulating the localization and activity of the transcriptional coactivator Yap1. Sci. Signal. 4:ra33.

Spagnuolo, R., M. Corada, F. Orsenigo, L. Zanetta, U. Deuschle, P. Sandy, C. Schneider, C.J. Drake, F. Breviario, and E. Dejana. 2004. Gas1 is induced by VE-cadherin and vascular endothelial growth factor and inhibits endothelial cell apoptosis. Blood. 103:3005-3012. http://dx.doi .org/10.1182/blood-2003-07-2459

Taddei, A., C. Giampietro, A. Conti, F. Orsenigo, F. Breviario, V. Pirazzoli, M. Potente, C. Daly, S. Dimmeler, and E. Dejana. 2008. Endothelia adherens junctions control tight junctions by VE-cadherin-mediated upregulation of claudin-5. Nat. Cell Biol. 10:923-934. http://dx.doi.org /10.1038/ncb1752

Taylor, M.J., E.R. Tiangga, R.N. Mhuircheartaigh, and P.J. Cowen. 2012 Lack of effect of ketamine on cortical glutamate and glutamine in healthy volunteers: A proton magnetic resonance spectroscopy study. J. Psychopharmacol. (Oxford). 26:733-737. http://dx.doi.org/10.1177 /0269881111405359

Tocchetti, A., C.B. Soppo, F. Zani, F. Bianchi, M.C. Gagliani, B. Pozzi, J. Rozman, R. Elvert, N. Ehrhardt, B. Rathkolb, et al. 2010. Loss of the actin remodeler Eps8 causes intestinal defects and improved metabolic status in mice. PLoS One. 5:e9468. http://dx.doi.org/10.1371/journal .pone.0009468

Vandenbroucke, E., D. Mehta, R. Minshall, and A.B. Malik. 2008. Regulation of endothelial junctional permeability. Ann. N.Y. Acad. Sci. 1123:134-145. http://dx.doi.org/10.1196/annals.1420.016

Varelas, X. 2014. The Hippo pathway effectors TAZ and YAP in development, homeostasis and disease. Development. 141:1614-1626. http://dx.doi.org /10.1242/dev.102376

Vestweber, D., M. Winderlich, G. Cagna, and A.F. Nottebaum. 2009. Cell adhesion dynamics at endothelial junctions: VE-cadherin as a major player. Trends Cell Biol. 19:8-15. http://dx.doi.org/10.1016/j.tcb.2008.10.001

Vestweber, D., A. Broermann, and D. Schulte. 2010. Control of endothelial barrier function by regulating vascular endothelial-cadherin. Curr. Opin. Hematol. 17:230-236. http://dx.doi.org/10.1097/MOH.0b013e328338664b

Zhao, B., X. Wei, W. Li, R.S. Udan, Q. Yang, J. Kim, J. Xie, T. Ikenoue, J. Yu, L. Li, et al. 2007. Inactivation of YAP oncoprotein by the Hippo pathway is involved in cell contact inhibition and tissue growth control. Genes Dev. 21:2747-2761. http://dx.doi.org/10.1101/gad.1602907

Zhao, B., K. Tumaneng, and K.L. Guan. 2011. The Hippo pathway in organ size control, tissue regeneration and stem cell self-renewal. Nat. Cell Biol. 13:877-883. http://dx.doi.org/10.1038/ncb2303 
Giampietro et al., http://www.jcb.org/cgi/content/full/jcb.201501089/DC1

A
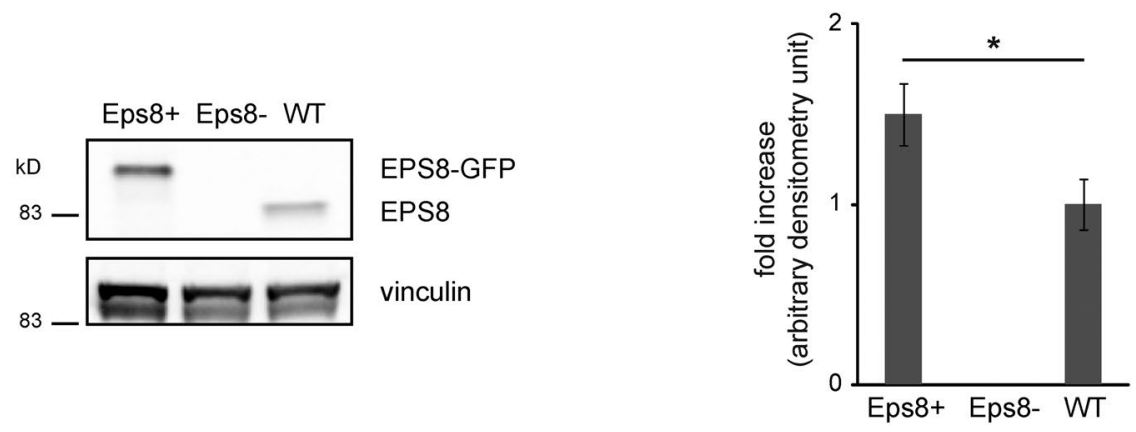

$\mathbf{B}$
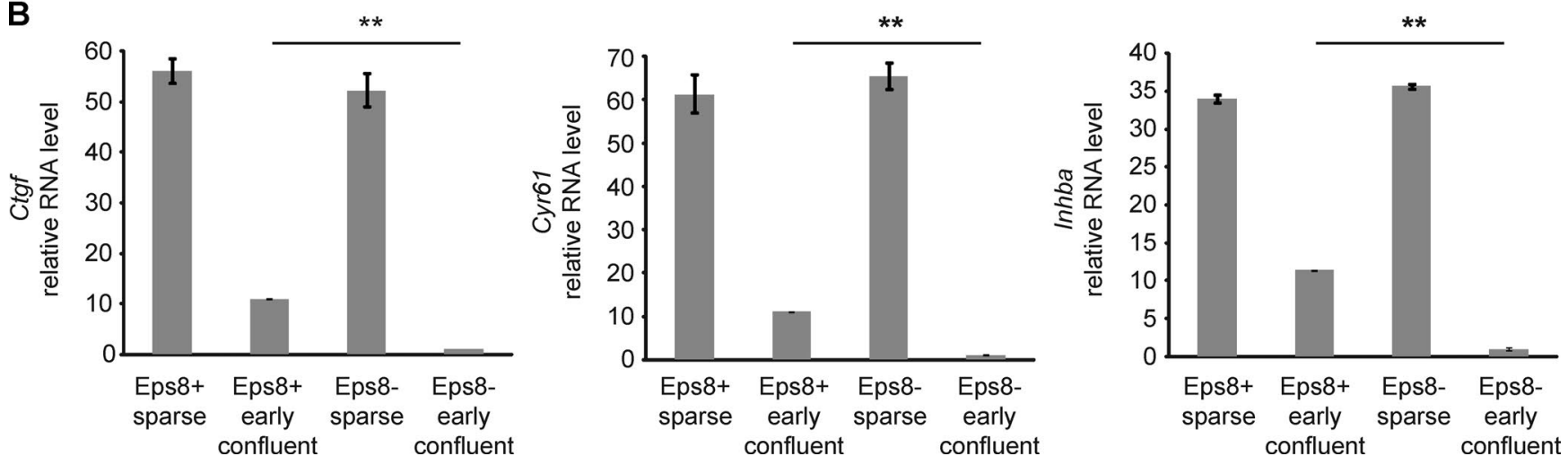

Figure S1. EPS8 reconstitution in EPS8- ECs. (A) WB analysis of EPS8 expression levels in EPS8+ and WT lung-derived ECs (left). Vinculin is shown as a loading control. Quantification of three independent experiments (right) is shown as means \pm SEM. *,$P<0.05$. (B) qRT-PCR analysis of Ctgf, Cyr6 1, and Inhba in EPS8 ${ }^{+}$and EPS8- ECs in sparse and confluent conditions. For each tested gene, the expression level has been represented as fold changes \pm SEM of three independent experiments. ${ }^{*}, \mathrm{P}<0.01$.
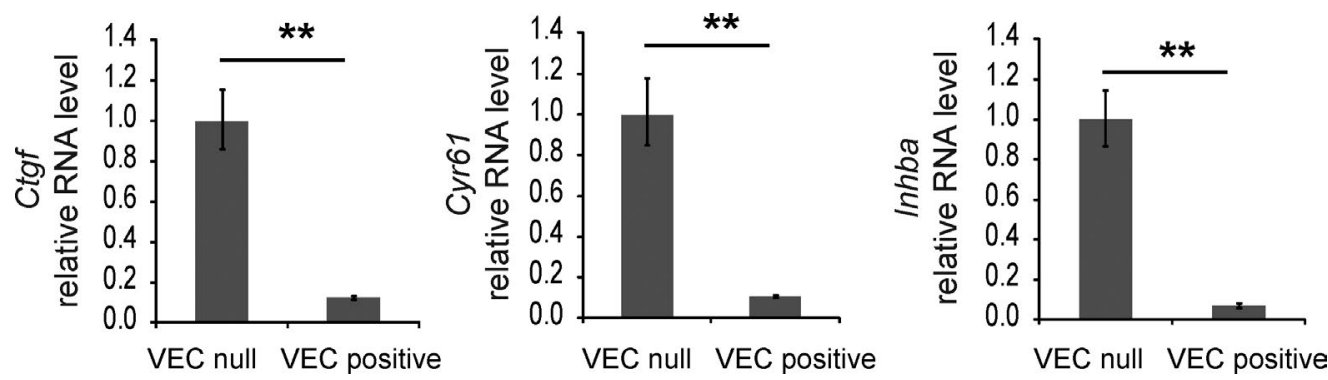

Figure S2. YAP transcriptional activity is regulated by VE-cadherin. qRT-PCR analysis of Ctgf, Cyr61, and Inhba in VE-cadherin-null and VE-cadherinpositive ECs to measure YAP transcriptional activity. The expression of VE-cadherin strongly inhibited YAP transcriptional activity. For each tested gene, the expression level has been represented as fold changes \pm SEM of four independent experiments. ${ }^{* *}, P<0.01$. VEC, VE-cadherin. 

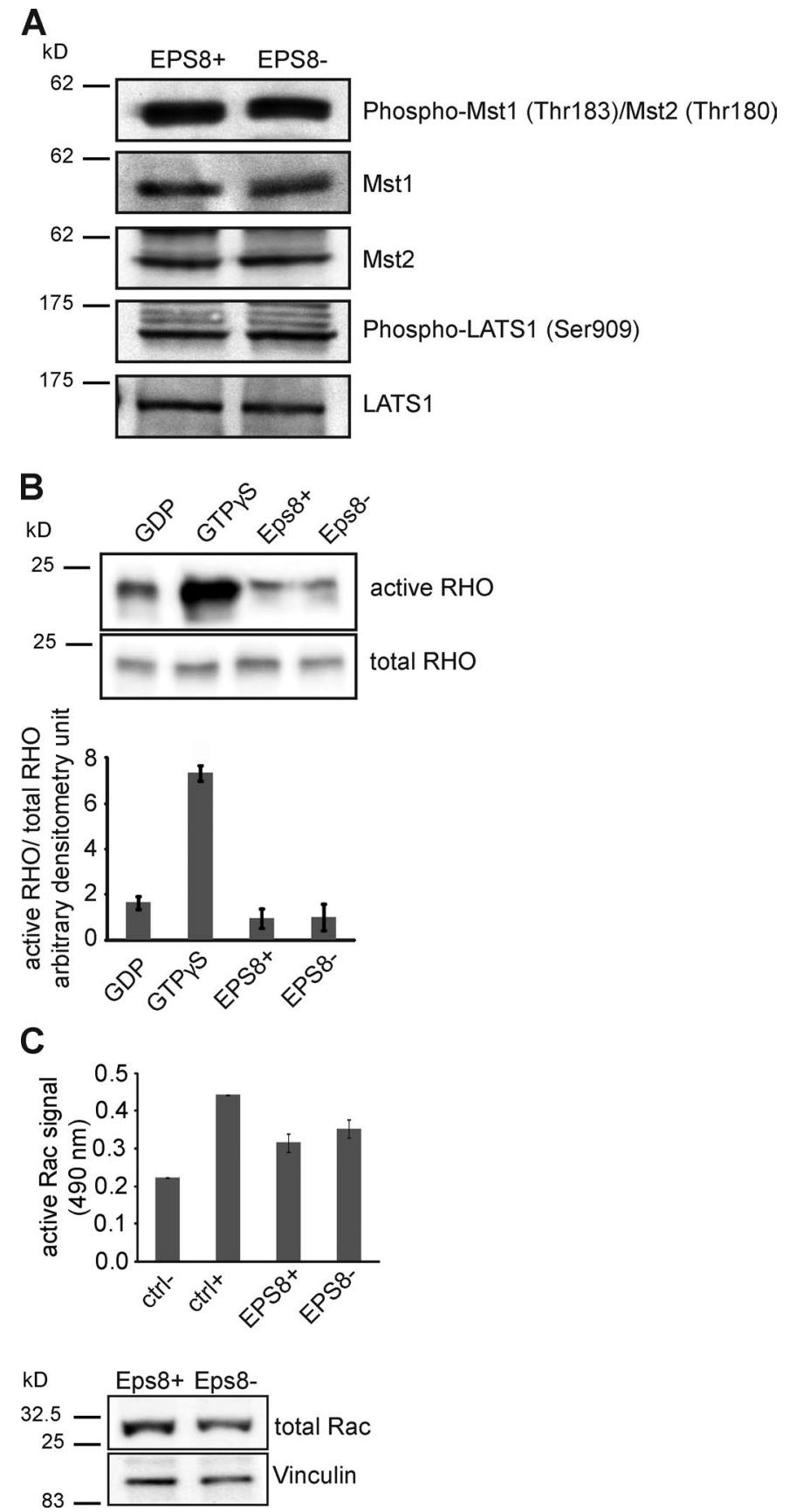

Figure S3. EPS8 expression does not influence the Hippo pathway signaling and RHO and RAC activity in ECs. (A) WB analysis of the Hippo pathway activation. Mst1, Mst2, and LATS1 phosphorylation (Phosho-Mst1 threonine 183, Phospho-Mst2 threonine 180, and Phospho-LATS1 serine 909, respectively) in confluent EPS8+ and Eps8- ECs. (B) Analysis of the Rho GTPase activity by pull-down assay (top) and quantification (bottom) in confluent EPS8+ and EPS8- ECs. (C) Analysis of the Rac activation assay (top) in confluent EPS8+ and EPS8- ECs. WB analysis of the total Rac expression levels (bottom). Vinculin is shown as a loading control. 
A

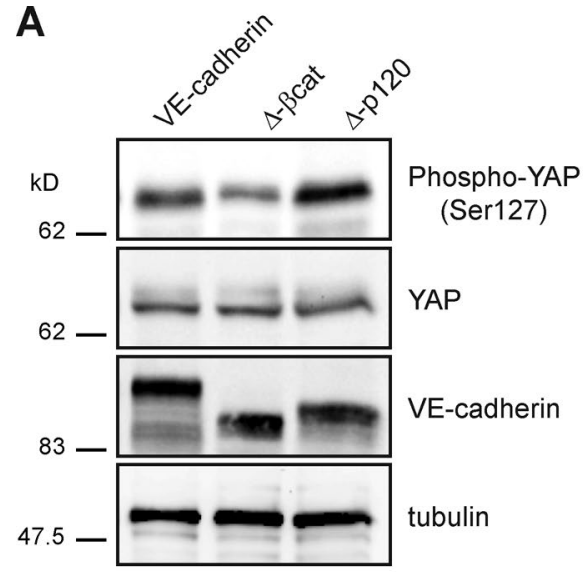

B

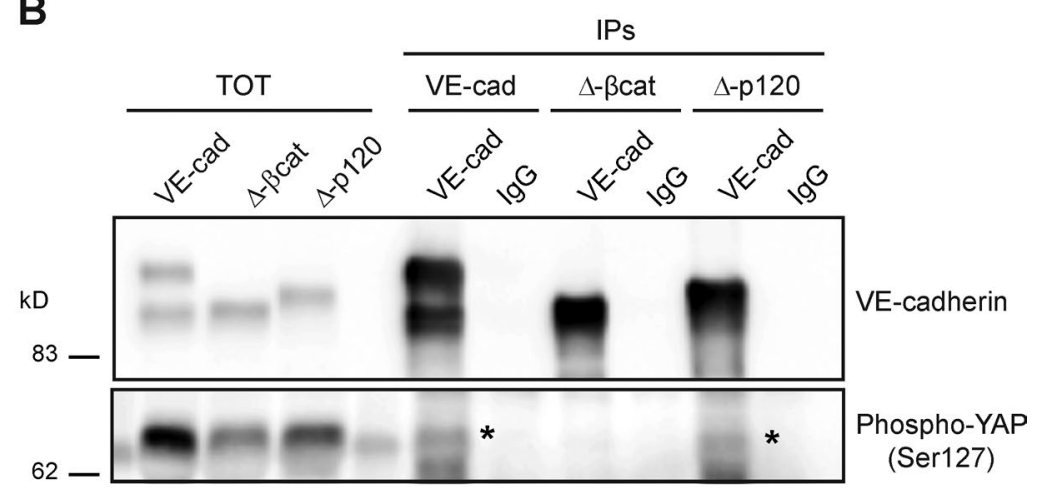

C

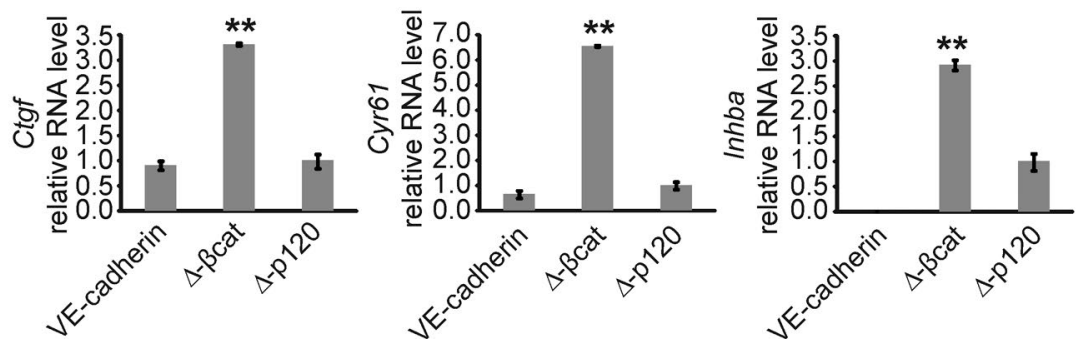

Figure S4. YAP binding to VE-cadherin negatively regulates its transcriptional activity. (A) WB analysis of YAP phosphorylation in ECs expressing fulllength (VE-cadherin positive), $\Delta$ - $\beta$ cat, and $\Delta$-p 120 mutants of VE-cadherin. The level of phosphorylation of YAP is reduced in $\Delta-\beta$ cat cells compared with that of the other cell lines. (B) IP and WB analysis of the VE-cadherin-Phospho-YAP complex in ECs expressing full-length (VE-cadherin positive), $\Delta$ - $\beta$ cat, and $\Delta$-p120 mutants of VE-cadherin. $\Delta$ - $\beta$ cat VE-cadherin did not bind Phospho-YAP (asterisks). (C) qRT-PCR analysis of Ctgf, Cyrb 1, and Inhba to measure YAP transcriptional activity in ECs expressing full-length (VE-cadherin positive), $\Delta$ - $\beta$ cat, and $\Delta$-p 120 mutants of VE-cadherin. In $\Delta$ - $\beta$ cat cells, YAP transcriptional activity is strongly increased compared with that of the other cell lines. For each tested gene, the expression level has been represented as fold changes \pm SEM of three independent experiments. ${ }^{*}, \mathrm{P}<0.01$.

WT
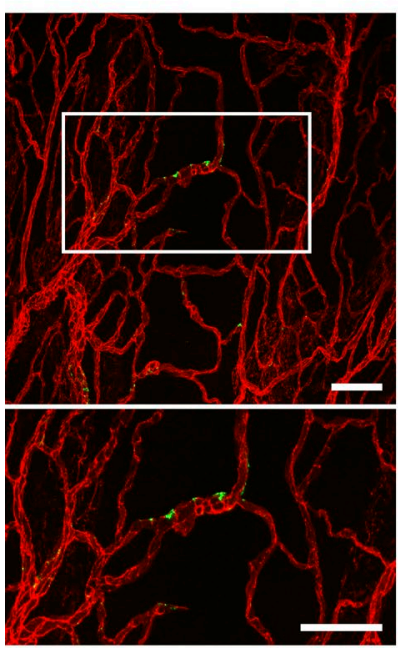

Microspheres Pecam-1 eps8 null
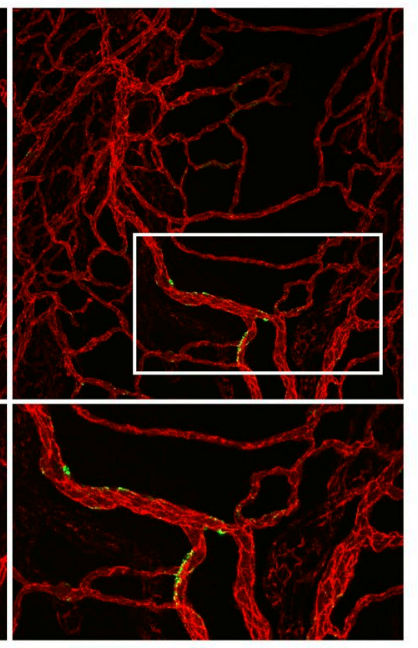

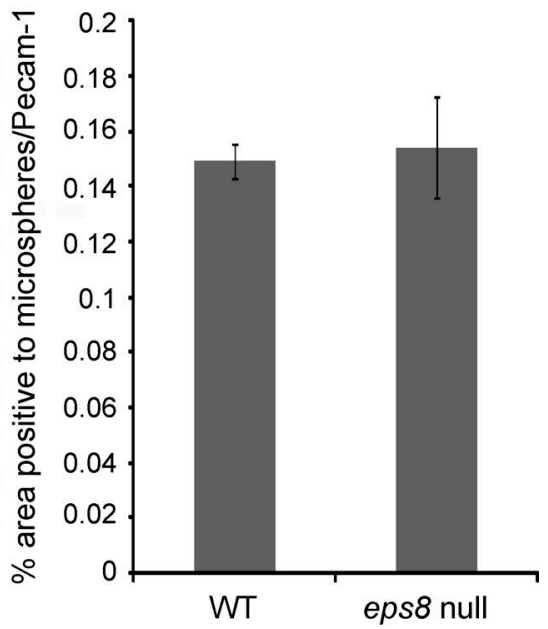

Figure S5. The absence of EPS8 does not impair the correct control of permeability to high-size tracer in vivo. In vivo permeability assay. 2 min before fixation, green fluorescent microspheres were intravenously injected. Permeability of the trachea vasculature (Pecam-1; red) has been measured by fluorescent microsphere extravasation (green). Bars, $100 \mu \mathrm{m}$. Data are means \pm SEM of three WT and three eps8-null mice. A white box indicates the area shown in the magnification below. $\mathrm{P}=0.54$. 